\title{
Chronic mineral oil administration increases hepatic inflammation in wild type mice compared to lipocalin
}

\section{2 null mice}

\author{
Erawan Borkham-Kamphorst ${ }^{1 凶}$, Ute Haas ${ }^{1}$, Manuela Pinoé-Schmidt ${ }^{1}$, Ali T. Abdallah ${ }^{2}$ and Ralf Weiskirchen (iD) ${ }^{1 凶}$ \\ (c) The Author(s) 2021
}

\begin{abstract}
Lipocalin 2 (LCN2), an acute-phase protein produced during acute liver injury, plays an important role in the innate immune response against bacterial infection via iron scavenging. LCN2 further influences neutrophil development and physiology leading to increased inflammatory responses. We investigated the roles of LCN2 in chronic inflammation and fibrosis, using repeated carbon tetrachloride $\left(\mathrm{CCl}_{4}\right)$ in mineral-oil injection. Surprisingly, mice treated with the mineral oil vehicle alone showed liver inflammation, evidenced by neutrophil and monocyte-macrophage infiltration. Fluorescence-activated cell sorting (FACS) of isolated liver leukocytes showed significantly high $\mathrm{CD}_{4} 5^{+}$leukocyte concentrations in $\mathrm{CCl}_{4}$ mice, but no difference of $\mathrm{Ly}_{6 \mathrm{G}}{ }^{+}$neutrophils between mineral oil and $\mathrm{CCl}_{4}$ application. Liver $\mathrm{CD} 11 \mathrm{~b}^{+} \mathrm{F} 4 / 80^{+}$cells counted higher in $\mathrm{CCl}_{4}$ mice, but the proportions of $\mathrm{Gr} 1^{\text {high }}$, an indicator of inflammation, were significantly higher in mineral oil groups. Liver myeloperoxidase (MPO), expressed in neutrophils and monocytes, showed higher levels in wild type mice compared to $\mathrm{LCn}^{-1-}$ in both mineral-oil and $\mathrm{CCl}_{4}$ treated groups. Hepatic and serum LCN2 levels were remarkably higher in the mineral oil-injected wild type group compared to the $\mathrm{CCl}_{4}$. Wild type animals receiving mineral oil showed significantly higher inflammatory cytokine- and chemokine mRNA levels compared to $L c n 2^{-/-}$mice, with no differences in the $\mathrm{CCl}_{4}$ treated groups. RNA sequencing (RNA-Seq) confirmed significant downregulation of gene sets involved in myeloid cell activation and immune responses in Lcn2 null mice receiving chronic mineral oil versus wild-type. We observed significant upregulation of gene sets and proteins involved in cell cycle DNA replication, with downregulation of collagencontaining extracellular matrix genes in $\mathrm{LCn}^{-1-}$ mice receiving $\mathrm{CCl}_{4}$, compared to the wild type. Consequently, the wild type mice developed slightly more liver fibrosis compared to $\mathrm{Lcn}^{-/-}$mice, evidenced by higher levels of collagen type I in the CCl $\mathrm{groups}$ and no liver fibrosis in mineral oil-treated mice. Our findings indicate that serum and hepatic LCN2 levels correlate with hepatic inflammation rather than fibrosis.
\end{abstract}

Laboratory Investigation (2021) 101:1528-1539; https://doi.org/10.1038/s41374-021-00672-9

\section{INTRODUCTION}

Lipocalin 2 (LCN2) or neutrophil gelatinase-associated lipocalin (NGAL) in human is a $24 \mathrm{kDa}$ secretory glycoprotein, isolated from specific granules of neutrophils ${ }^{1}$. LCN2 plays an important role in innate immune responses against bacterial infections by sequestering iron-containing siderophores ${ }^{2,3}$. Consequently, Lcn2 knockout $\left(\operatorname{LCn} 2^{-/-}\right)$mice are susceptible to infections, especially to siderophore-producing bacteria ${ }^{4}$. In $\operatorname{Lcn} 2^{-/-}$mice neutrophil maturation and functionality are affected, which is reflected by increased band cells in circulation and higher amounts of neutrophil count and reduced migratory activity, adhesion, tissue extravasation, phagocytosis, and chemotaxis ${ }^{5}$. Lcn2 ${ }^{-1-}$ mice, therefore, are sensitive to pathogens, in part due to impaired functioning in neutrophils ${ }^{5-7}$. LCN2 induces the production of cytokines and chemokines to promote the migration and phagocytosis of macrophages, resulting in stronger inflammatory responses in wild type compared to $\operatorname{Lcn} 2^{-1-}$ mice ${ }^{6,7}$. However, in non-pathogen-driven inflammatory conditions such as non-alcoholic steatohepatitis (NASH) and alcoholic steatohepatitis (ASH), LCN2 plays detrimental roles in the liver through neutrophil infiltration ${ }^{8-10}$. On the contrary, LCN2 showed protective effects in bacterial infections and animal models of liver injury ${ }^{11-15}$. Furthermore, human serum LCN2 is a biomarker of acute-onchronic liver failure and prognosis in cirrhosis ${ }^{16}$ and reported to correlate to non-alcoholic fatty liver disease (NAFLD) ${ }^{17}, \mathrm{ASH}$ disease severity, and the model for end-stage liver disease (MELD) score $^{18}$. We and others did not find systemic LCN2 levels to directly correlate to impaired liver function and disease severity $^{15,19}$. We here used the 8 week-repeated carbon tetrachloride $\left(\mathrm{CCl}_{4}\right)$ intraperitoneal injections of female C57BL/6 wild type and $\mathrm{LCn} 2^{-/-}$mice to evaluate the role of LCN2 in hepatic inflammation and fibrosis. Unexpectedly, mice that received the mineral oil vehicle showed liver inflammation as evidenced by neutrophil and monocyte-macrophage infiltration. Fluorescence-activated cell sorting (FACS) of isolated liver leukocytes revealed significantly higher quantities of liver $\mathrm{CD}_{4} 5^{+}$leukocytes in $\mathrm{CCl}_{4}$ mice, with no

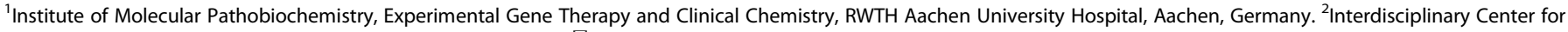
Clinical Research, University Hospital RWTH, Aachen, Germany. ${ }^{凶}$ email: eborkham@ukaachen.de; rweiskirchen@ukaachen.de 
marked differences of $\mathrm{Ly}_{6 \mathrm{G}}{ }^{+}$neutrophils in the mineral oil- and $\mathrm{CCl}_{4}$-treated groups. Levels of liver $\mathrm{CD} 11 \mathrm{~b}^{+} \mathrm{F} 4 / 80$ cells were higher in $\mathrm{CCl}_{4}$ mice, but the proportions of the inflammation indicator $\mathrm{Gr} 1^{\text {high }}$, were significantly higher in the mineral oil groups. Hepatic expression of myeloperoxidase (MPO), the proinflammatory enzyme expressed in neutrophils and monocytes, was found to be higher in wild type mice as compared to $\operatorname{Lcn} 2^{-/-}$ mice after both mineral oil and $\mathrm{CCl}_{4}$ administration. The levels of hepatic and serum LCN2 were significantly higher in the mineral oil-injected wild type group relative to $\mathrm{CCl}_{4}$. Wild type animals receiving mineral oil showed significantly higher levels of inflammatory cytokine- and chemokine mRNA compared to $\mathrm{LCn} 2^{-1-}$ mice, while the expression in the $\mathrm{CCl}_{4}$ groups was similar. RNA sequencing (RNA-Seq) showed significant downregulation of gene sets involved in myeloid cell activation and immune responses in Lcn2 null mice treated with mineral oil versus wild type. Surprisingly, we noted significant upregulation of gene sets and proteins involved in DNA replication and cell cycle and downregulation of collagen-containing extracellular matrix genes in $\mathrm{LCn} 2^{-1-}$ mice receiving $\mathrm{CCl}_{4}$, compared to the wild type. Consequently, $\mathrm{CCl}_{4}$ administered wild type mice developed slightly more liver fibrosis than $L c n 2^{-1-}$ mice, evidenced by higher levels of collagen type I in the $\mathrm{CCl}_{4}$ groups. No significant liver fibrosis was observed in mice treated with mineral oil.

\section{MATERIALS AND METHODS}

\section{Animal experiments and specimen collections}

Eight-week-old female C57BL/6 wild type and Lcn2 $2^{-1-}$ mice $^{4}$ were subjected to intraperitoneal (i.p.) injection of $0.8 \mathrm{ml} / \mathrm{kg}$ body weight $\mathrm{CCl}_{4}$ diluted in mineral oil twice weekly for 8 weeks. The mineral oil vehicle was applied to control animals. Mice were sacrificed 48 hours upon the last injection, heart blood samples were collected followed by liver perfusion. Liver specimens were snap-frozen in liquid nitrogen and stored at $-80^{\circ} \mathrm{C}$ for protein and RNA isolation. Tissue frozen sections and paraffinembedded sections were prepared for histology. All animal protocols complied with the guidelines for animal care approved by the German Animal Care Committee (Landesamt für Naturschutz, Umwelt und Verbraucherschutz Nordrhein-Westfalen (LANUV) located in Recklinghausen, Germany; https://www.lanuv.nrw.de).

\section{Intrahepatic leukocyte isolations}

Most of the liver tissue was used for intrahepatic leukocyte isolation and flow cytometric analysis as described ${ }^{20}$. In brief, livers were perfused with phosphate-buffered saline (PBS), minced with scissors, and subsequently digested for $30 \mathrm{~min}$ at $37^{\circ} \mathrm{C}$ with collagenase type-IV (Worthington Biochemical, NJ, USA). To gain single-cell suspensions, the digested products were pressed through $70-\mu \mathrm{m}$ cell strainers. A small aliquot was taken to stain with CD45 for assessment of the relative number of intrahepatic leukocytes $\left(\mathrm{CD} 45^{+}\right)$to all liver cells. The remaining liver singlecell suspension was subjected to density gradient centrifugation in Lymphocyte Separation Medium (LSM-1077, PAA Laboratories, Cölbe, Germany) at $2000 \mathrm{rpm}$ for $20 \mathrm{~min}$ at $25^{\circ} \mathrm{C}$. Upon centrifugation, the leukocytes were collected from the interphase. Cells were washed twice with Hank's balanced salt solution containing $2 \%$ bovine serum albumin and $0.1 \mathrm{mM}$ ethylenediaminetetraacetic acid (EDTA) and subjected to FACS.

\section{Flow cytometry}

Six-color staining was performed using combinations of the following monoclonal antibodies: F4/80 (Serotec, Puchheim, Germany), CD115, CD4, CD11b (all from eBioscience, Frankfurt/Main, Germany), CD45, Gr1/Ly6C, Ly6G, NK, CD8, CD3, mouse immunoglobulin (lg) G1, rat lgG2a, or hamster lgG isotype controls (all BD Bioscience, Heidelberg, Germany). Flow cytometric analysis was performed on a FACS-Canto (BD) and analyzed with FlowJo (Tree Star Inc., Ashland, OR, USA).

\section{RNA isolation, CDNA synthesis, and RT-qPCR}

Liver tissue was homogenized in QIAzol lysis reagent (Qiagen, Hilden, Germany) in a Mixer Mill MM400 homogenizer (Retsch, Haan, Germany).
Total RNA was isolated as described previously through phenol-chloroform extraction, isopropanol precipitation, and DNAse digestion followed by subsequent RNA clean up with PureLink RNA Mini kits (Invitrogen, Thermo Fisher Scientific, Darmstadt, Germany) according to manufacturer's guidelines $^{21}$. Primers for amplification were selected from the sequences deposited in the GenBank database using the online ProbeFinder Software (Universal Probe Library Assay Design Center, Roche, Mannheim, Germany). First-strand cDNA was synthesized from $1 \mu \mathrm{g}$ RNA in $20 \mu \mathrm{l}$ volume using SuperScript ${ }^{\mathrm{TM}}$ II reverse transcriptase and random hexamer primers (Invitrogen). For quantitative real-time PCR (RT-qPCR), CDNA derived from $50 \mathrm{ng}$ RNA ( $5 \mu \mathrm{l}$ of 1:5 dilution of CDNA) was amplified in $25 \mu \mathrm{l}$ volume using SYBR ${ }^{\circ}$ GreenER ${ }^{\text {TM }}$ qPCR SuperMix for ABI PRISM ${ }^{\oplus}$ (Invitrogen). The PCR conditions were set to $50^{\circ} \mathrm{C}$ for $2 \mathrm{~min}, 95^{\circ} \mathrm{C}$ for $10 \mathrm{~min}$ initial denaturation, followed by 40 cycles of $95^{\circ} \mathrm{C}$ for $15 \mathrm{sec}$, and $60^{\circ} \mathrm{C}$ for $1 \mathrm{~min}$. Relative mRNA expression was normalized to the housekeeping gene glyceraldehyde 3-phosphate dehydrogenase $(G A P D H)$ and calculated using $2^{-\Delta \Delta C T}$ method $^{22}$. All primers used in this study are given in Supplementary Table 1.

\section{RNA-Seq library preparation and sequencing}

Quality checks of RNA samples were performed with the TapeStation 4200 using the Agilent RNA ScreenTape assay kit (Agilent, Waldbronn, Germany). Quantification was performed using the Quantus RNA System (Promega, Madison, WI, USA). According to the manufacturer's instructions, CDNA libraries were produced employing the rapid MACE kit for $3^{\prime}$ end RNA Sequencing (GenXPro GmbH, Frankfurt am Main, Germany), using $100 \mathrm{ng}$ input RNA. $20 \%(260 \mu \mathrm{l})$ of denatured and diluted PhiX was added to the final loading mixture to increase the mixture's diversity and enhance the kit's performance. The quality and quantity of the cDNA libraries were assessed using the 4200 TapeStation (D1000 screen tape assay) and the Quantus dsDNA system. All samples were sequenced on a NextSeq 500 sequencing system with the following run parameters: 76 cycles for read 1, 6 cycles for index 1 . The cDNA libraries were generated using a TruSeq Stranded Total RNA Sample Preparation Kit (Illumina, San Diego, CA, USA).

\section{RNA-Seq primary analysis}

Generation of the fastq files and removal of adapters were completed using the Illumina demultiplexing and conversion software bcl2fasta (https://support.illumina.com/sequencing/sequencing_software/bcl2fastqconversion-software.html). Subsequently, RNA-Seq primary analysis was performed with the RNA-Seq pipeline of nf.core v3.23 using standard parameters. For instance, quality control of the sequenced transcripts was done with online routine fastqc v0.11.9 (http://www.bioinformatics. babraham.ac.uk/projects/fastqc), library complexity was measured with preseq $v 2 \cdot 0.3^{24}$. Adapter and quality trimming was performed using TrimGalore v.0.6.6 (https://www.bioinformatics.babraham.ac.uk/projects/ trim_galore/). Subsequently, the reads were aligned to the $\mathrm{mm} 10$ reference genome sequence using STAR $\mathrm{v} 2.6 .1 \mathrm{~d}^{25}$ with default parameters. In the quantification step, reads were counted with the quasi-mapping quantification tool Salmon ${ }^{26}$.

\section{Differential expression analysis}

Differential expression analysis (DEA) was then performed in $\mathrm{R}$ using the DESeq function of the DESeq2 v1.30.0 package ${ }^{27}$, with local fit type (fitType $=$ "local"), without LFC modulation (betaPrior = False) and using the Wald test as significance test. Genes with log fold change $>0.56$ ( $F C \geq$ 1.5 ) and corrected $p$ value $\leq 0.05$ were considered as differentially expressed.

\section{GO enrichment analysis}

GO enrichment analyses (GOA) for each of the comparisons were performed with the gprofiler2 package v0.2. $0^{28}$, using the package's gost function, making an ordered query (genes sorted by significance) with an adjusted $p$-value threshold of 0.05 and the multiple test correction method gSCS.

\section{Availability of data and code}

For reproducibility, the script of the advanced analysis (DEA and GOA and various QC's and all plots used in this manuscript) is accessible via GitHub (https://github.com/ATA82/HepaticInflammation_LipocalinNullMice). The data are accessible via GEO accession number GSE176093. 
A

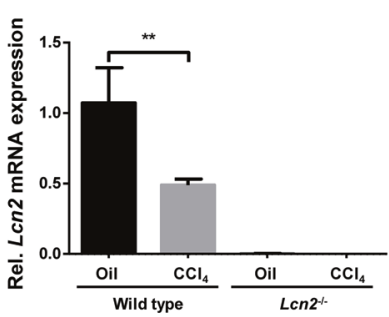

C
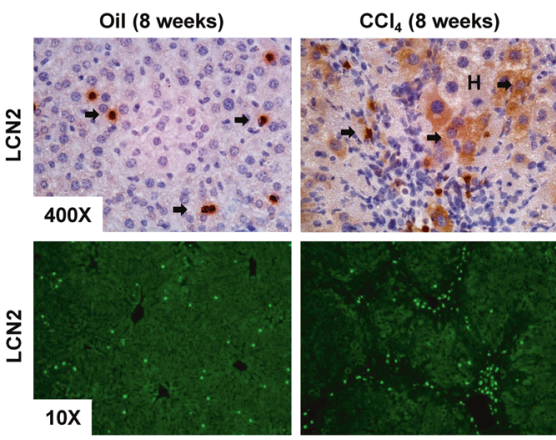

B
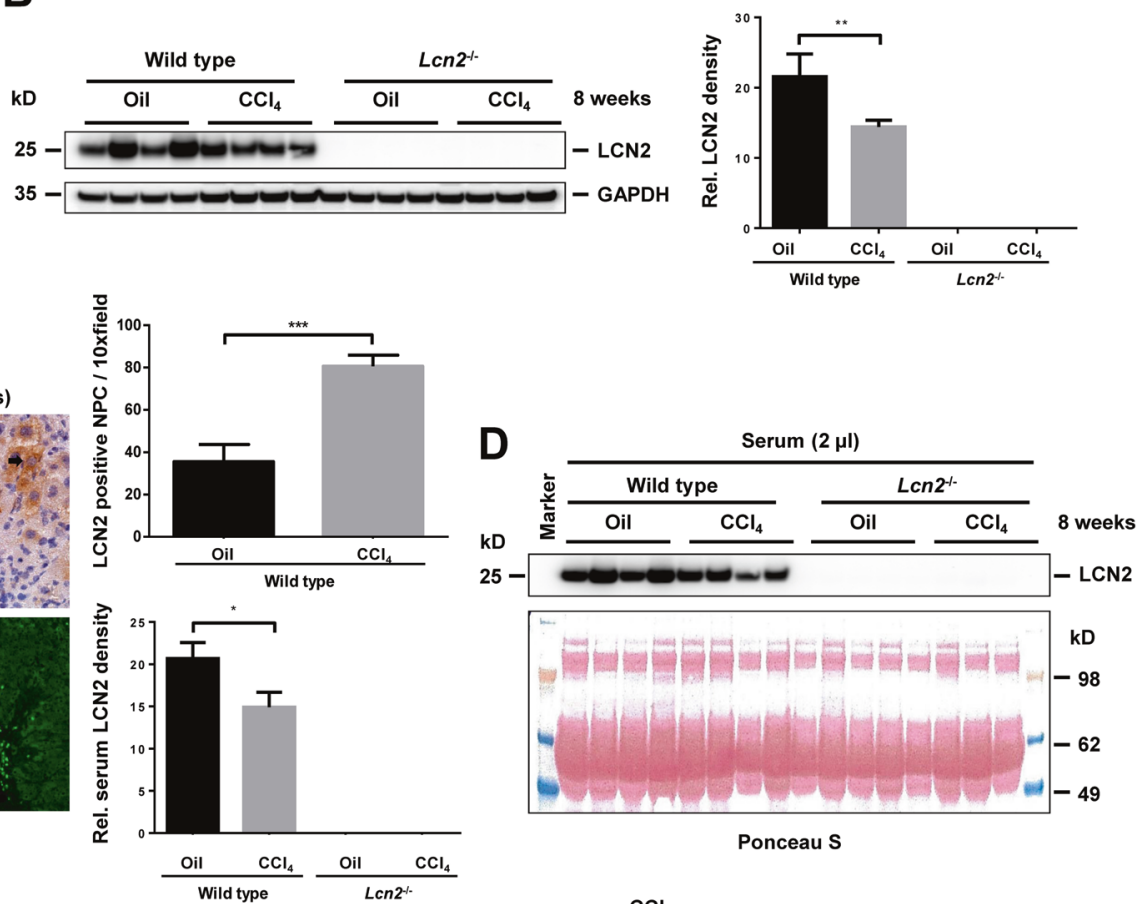

$\mathrm{E}$
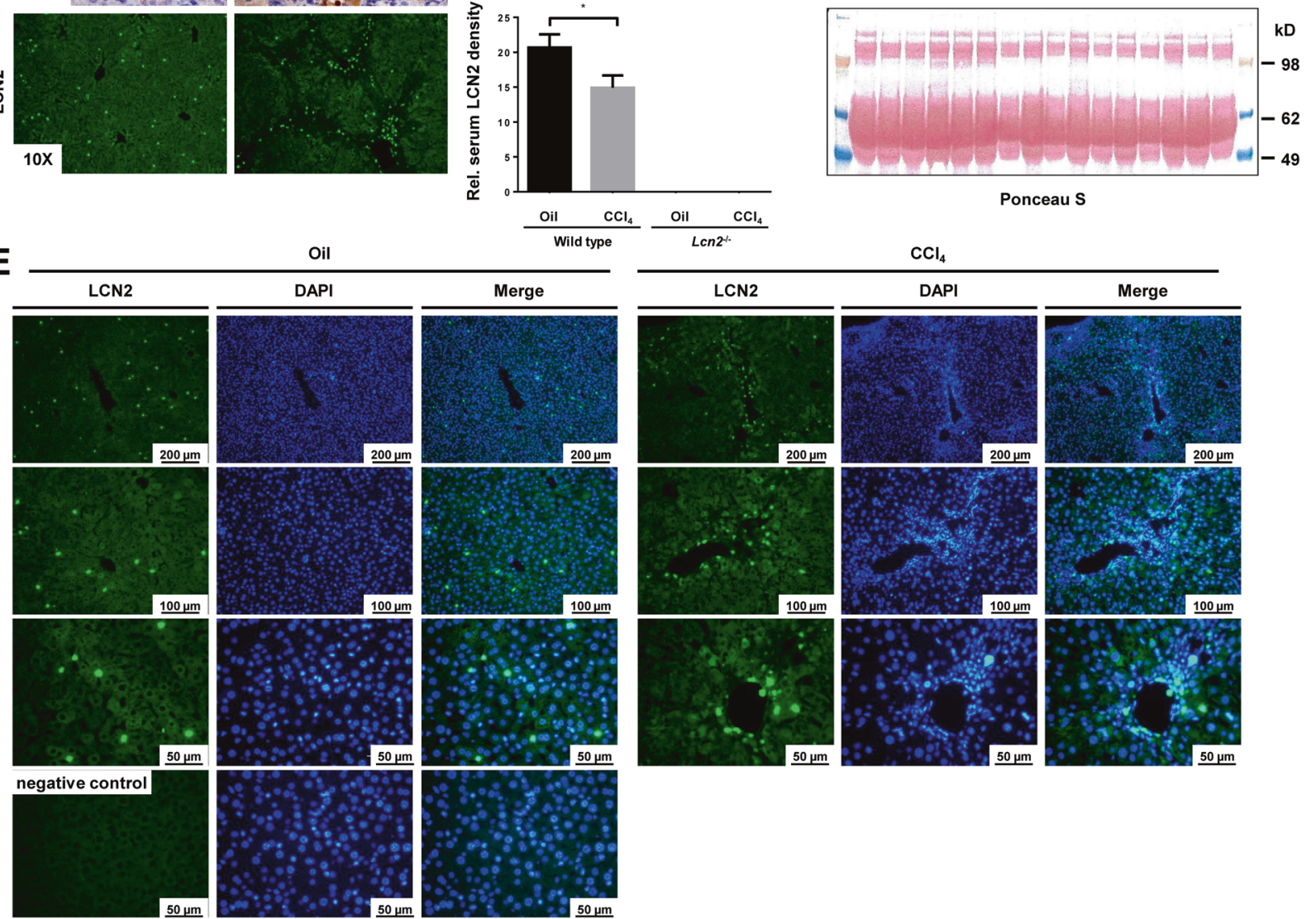

ceau S

Fig. 1 Long-term mineral oil and $\mathrm{CCl}_{4}$ induced liver LCN2 expression and increased serum LCN2. A Quantitative RT-PCR of Lcn2 shows significantly higher levels of hepatic Lcn2 in 8-week wild type oil administration compared to its $\mathrm{CCl}_{4}$ counterpart and no $L c n 2$ expression in $\mathrm{LCn}^{-\prime-}$ mice. B Western blot confirmed significantly higher amounts of LCN2 protein in wild type mice receiving oil than $\mathrm{CCl}_{4}$. C Liver IHC of LCN2 reflects LCN2 positive NPC scattered homogeneously in mineral oil injection, while $\mathrm{CCl}_{4}$ livers showed positive in both NPC infiltrating cells and hepatocytes around central veins. The LCN2 positive NPC are significantly higher in the $\mathrm{CCl}_{4}$ liver. D Western blot analysis of serum (2 $\mu \mathrm{l} /$ lane) also showed that LCN2 correlates well with liver mRNA and protein levels with significantly higher levels in oil-injected animals. E Liver tissue immunofluorescence staining from wild type animals found LCN2 positive hepatocytes and NPC in the liver of oil- and CCl ${ }_{4}^{-}$ treated groups.

\section{Liver protein lysate preparation, SDS-PAGE, and Western blot analysis}

Liver tissues were homogenized in a Mixer Mill MM400 homogenizer followed by ultrasound sonication in RIPA buffer containing $20 \mathrm{mM}$ Tris- $\mathrm{HCl}(\mathrm{pH} 7.2)$, $150 \mathrm{mM} \mathrm{NaCl}, 2 \%(\mathrm{w} / \mathrm{v}) \mathrm{NP}-40,0.1 \%(\mathrm{w} / \mathrm{v})$ SDS, 0.5\% (w/v) sodium deoxycholate, and the Complete ${ }^{\mathrm{TM}}$-mixture of proteinase inhibitors (Roche). Proteins were quantified using DC ${ }^{\text {m }}$ Protein assay kit I (Bio-Rad, Feldkirchen,
Germany) according to the manufacturer's instructions. Equal amounts of protein extracts $(50-100 \mu \mathrm{g})$ were diluted with Nu-PAGE ${ }^{\mathrm{M}}$ LDS electrophoresis sample buffer with $50 \mathrm{mM}$ dithiothreitol (DTT) as a reducing agent. The samples were heated at $95{ }^{\circ} \mathrm{C}$ for $10 \mathrm{~min}$ and separated in 4-12\% Bis-Tris gradient gels, using MES running buffers (all from Invitrogen). Proteins were electroblotted onto nitrocellulose membranes (Schleicher \& Schuell BioScience $\mathrm{GmbH}$, Dassel, Germany) and confirmed by Ponceau S staining. 
Non-specific binding sites were blocked with $5 \%(\mathrm{w} / \mathrm{v})$ non-fat milk powder in Tris-buffered saline and 0.1\% Tween 20 (TBST). All antibodies (Supplementary Table 2) were diluted in $2.5 \%(\mathrm{w} / \mathrm{v})$ non-fat milk powder in TBST. Primary antibodies were detected using horseradish peroxidase (HRP)-conjugated anti-mouse-, anti-rabbit-, or anti-goat lgG (Invitrogen) and the SuperSignal chemiluminescent substrate (Pierce, Bonn, Germany).

\section{Immunohistochemical and immunofluorescence staining}

Liver tissue sections were deparaffinized and rehydrated with xylene and decreasing graded ethanol, whereas antigen retrieval was performed by heating the sections in a $10 \mathrm{mM}$ sodium citrate buffer $(\mathrm{pH} 6.0)$ in a steamer for $30 \mathrm{~min}$. Tissue slides were blocked with avidin-biotin (DAKO, Hamburg, Germany), followed by incubation in a solution containing $2-5 \%$ animal serum of which the second antibody was made in $1 \%$ BSA and $0.05 \%$ Tween 20 in PBS (PBST). Primary antibodies were diluted in $1 \%$ BSA, $0.1 \%$ cold fish skin gelatin, $0.3 \%$ Triton X-100 in PBST to concentrations of $2-5 \mu \mathrm{g} / \mathrm{ml}$ and incubated at $4{ }^{\circ} \mathrm{C}$ overnight, while omitting the primary antibody was used for negative control. Nonspecific peroxidase blocking was performed with $3 \% \mathrm{H}_{2} \mathrm{O}_{2}$ for $10 \mathrm{~min}$. Sections were then incubated with biotinylated secondary antibodies (DAKO) for $1 \mathrm{~h}$, followed by avidin-biotin conjugated peroxidase for $30 \mathrm{~min}$. (VECTASTAIN ${ }^{\circledR}$ Elite ABC-HRP peroxidase kit (PK-6100), Vector Laboratories, Burlingame, CA, USA). The sections were developed using 3,3'-diaminobenzidine substrates (SIGMAFAST ${ }^{\mathrm{T}}$, Sigma-Aldrich, Taufkirchen, Germany), counterstained with hematoxylin (DAKO), and mounted with DPX Mountant for histology (Sigma-Aldrich).

For Immunofluorescence staining, liver tissue sections were prepared as mentioned above. The sections were blocked with $5 \%$ serum originating from the animal that the 2 nd antibody was made of in $1 \%$ BSA in PBST for $1 \mathrm{~h}$ at room temperature, followed by incubation of primary antibodies at

A

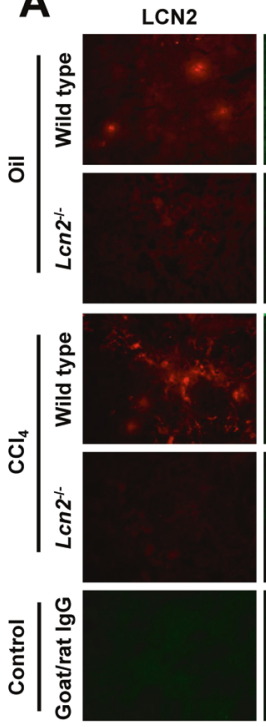

C
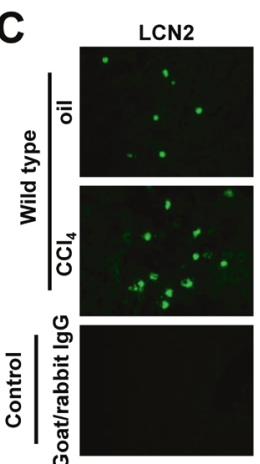

Ly6G
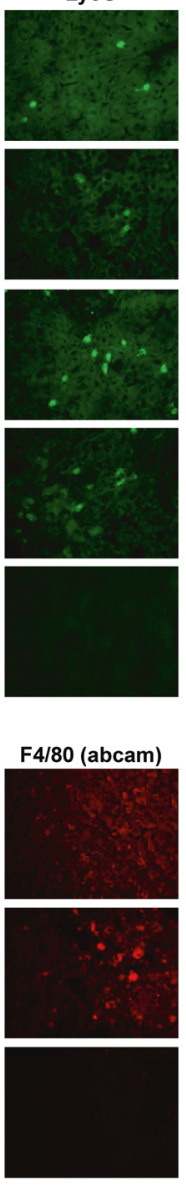

DAPI
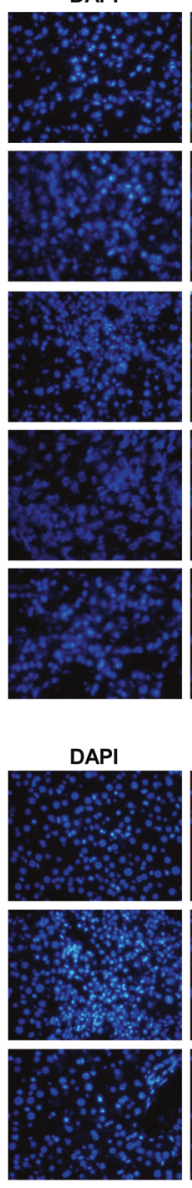

Merge
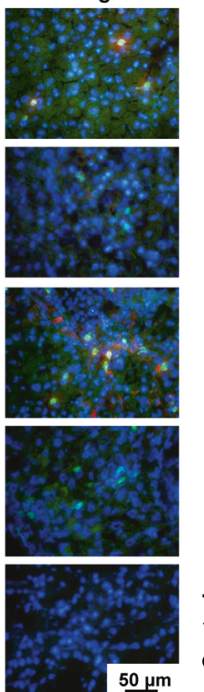

$50 \underline{\mu m}$

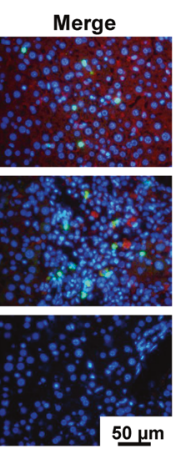

$4{ }^{\circ} \mathrm{C}$ overnight. Subsequently, the Alexa Fluor ${ }^{\circledast}$ conjugated secondary antibodies (Invitrogen) were applied for $1 \mathrm{~h}$ at room temperature and the nuclei were counterstained with 4',6-diamidino-2-phenylindole (DAPI). The tissue sections were mounted with coverslips using Permafluor mountant TA-030-FM (Thermo Fisher Scientific) and analyzed by fluorescence microscopy, using the Nikon Eclipse 80i microscope (Nikon, Tokyo, Japan), equipped with the NIS-Elements Vis software (version 3.22.01, Nikon, Amsterdam, The Netherlands).

\section{Statistics}

For statistical analysis we used GraphPad Prism 6 (GraphPad Software, Inc., San Diego, CA). Statistical significance of differences between mean values was determined using one-way analysis of variance (ANOVA) followed by Tukey Honestly Significant Difference (Tukey HSD) for multiple comparison tests. Probability values given are ${ }^{*} p<0.05,{ }^{* *} p<0.01$, and ${ }^{* * *} p<0.001$, respectively.

\section{RESULTS}

Long-term application of mineral oil and $\mathrm{CCl}_{4}$ induced hepatic LCN2 expression

Upon eight week- mineral oil or $\mathrm{CCl}_{4}$ administration, induction of hepatic LCn2 mRNA and protein levels was significant in wild type livers (Fig. 1A, B). Immunohistochemical staining showed LCN2 positive non-parenchymal cells (NPC) scattered homogeneously within the tissue after mineral oil administration, while $\mathrm{CCl}_{4}$ livers showed positive LCN2 in both non-parenchymal infiltrating cells and hepatocytes around central veins (Fig. 1C). LCN2 positive NPC

B

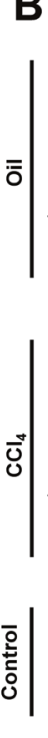

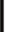

ड़
MPO
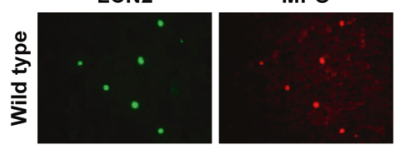

ปิ่
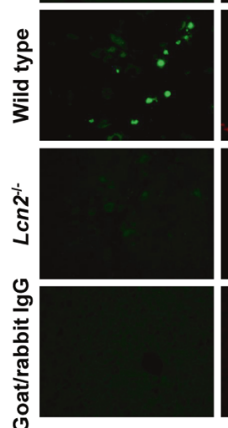

D

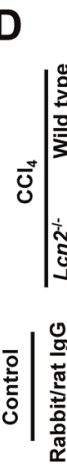

MPO
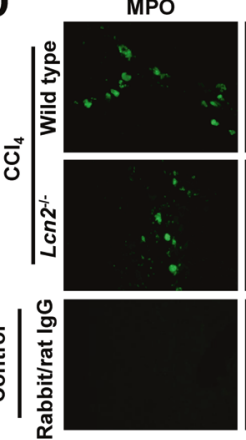
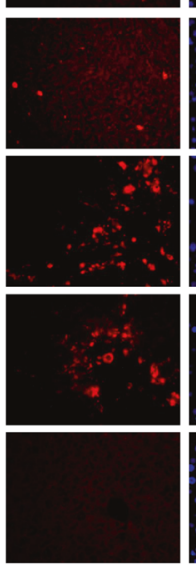

F4/80 (Serotec)

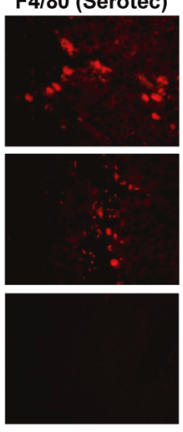

DAPI
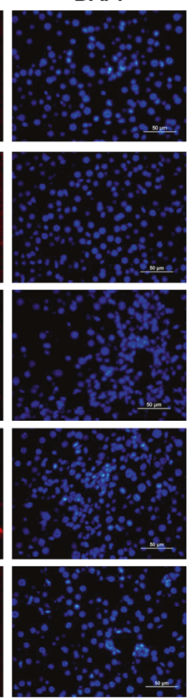

DAPI

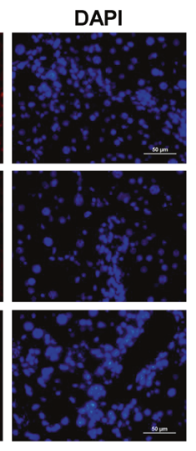

Merge
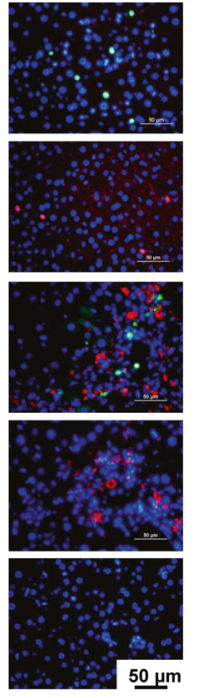

Merge

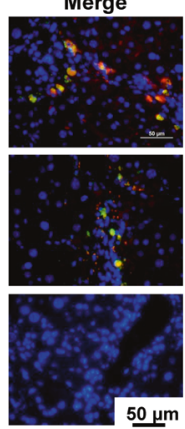

Fig. 2 Immunofluorescence double staining of liver sections. A Frozen section double staining of LCN2 (red) and Ly6G (green) shows colocalization of LCN2 and Ly6G. B Paraffin section staining of LCN2 (green) and MPO (red) showed co-localization of LCN2 and MPO in wild type oil administration but co-localized only partially in $\mathrm{CCl}_{4}$ around central veins. C No co-localization of LCN2 (green) and F4/80 (red) in both oil and $\mathrm{CCl}_{4}$ administration. $\mathbf{D ~ C C l}$ treated livers showed co-localization of MPO (green) and F4/80 (red) along the fibrotic central veins. 
A
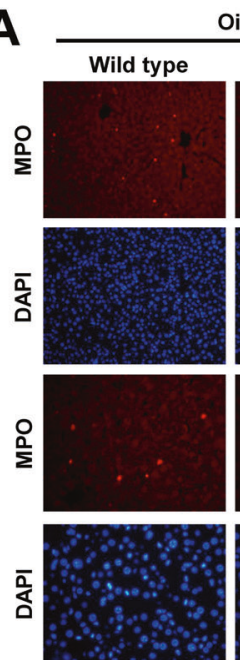

C
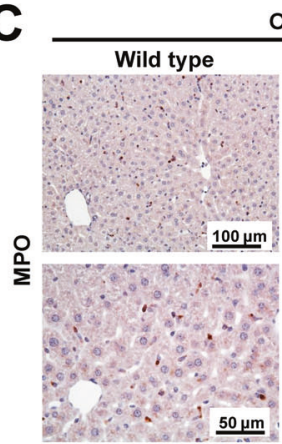

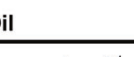
$\operatorname{Lcn} 2^{-1-}$
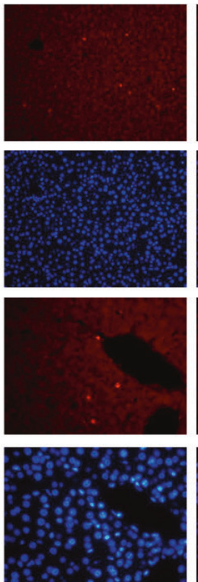

Oil Len $2^{-}$
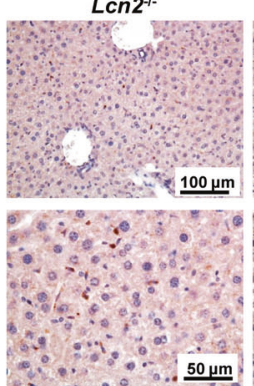

$\mathbf{E}$

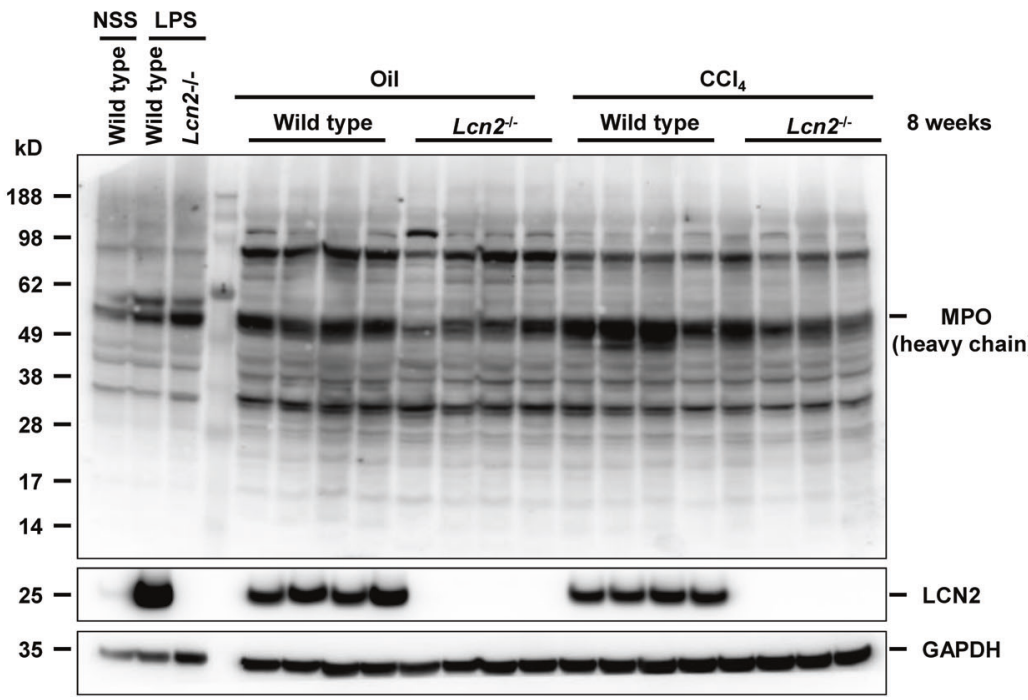

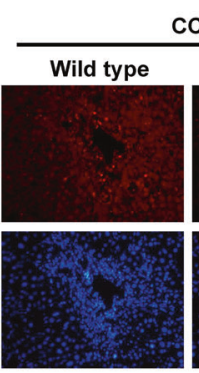
CC

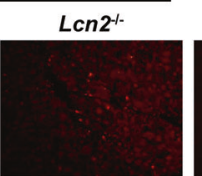

negative
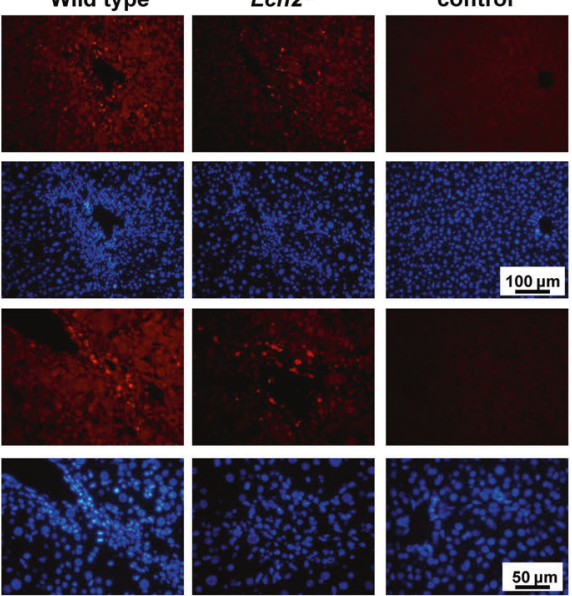

$\mathrm{CCl}_{4}$
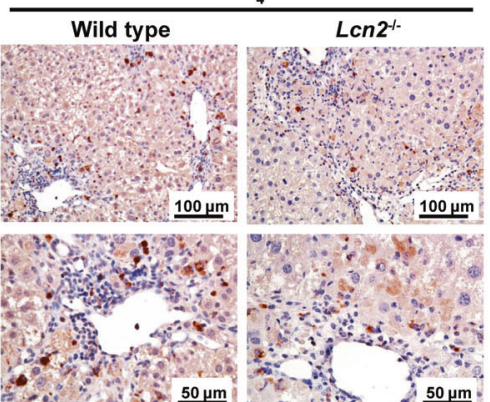

$\mathbf{F}$
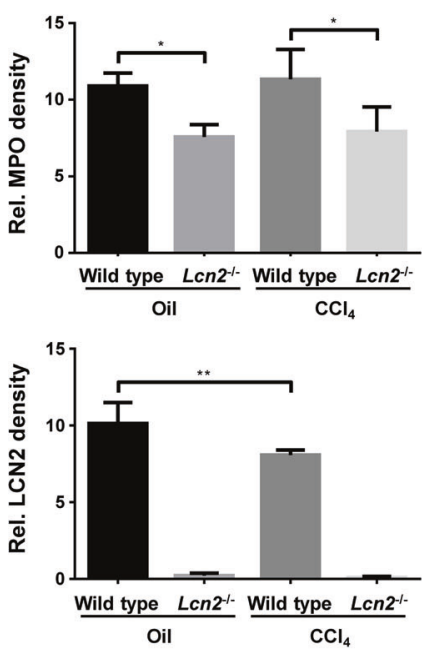

Fig. 3 Lcn2 null mice have decreased $\mathrm{MPO}^{+}$cells and MPO protein after mineral oil and $\mathrm{CCl}_{\mathbf{4}}$ administration. A IF staining shows lower numbers of $\mathrm{MPO}^{+}$cells in $\mathrm{LCn} 2^{-1-}$ receiving oil and $\mathrm{CCl}_{4}$ compared to the wild type. B The average cell counts of $\mathrm{MPO}^{+}$cells $/ 10 \times$ fields $(10$ fields/animal) were confirmed significantly lower in $\mathrm{Lcn}^{-1-}$ livers. C IHC staining of $\mathrm{MPO}^{+}$cells corresponds with immunofluorescence. D Western blot of HL-60 and NB4 cell lines incubated with LPS and tunicamycin (TM) were used as positive controls of the MPO antibody. The promyelocytic cell lines derived from human leukemia do not produce LCN2 upon LPS or LPS and TM application, HepG2 stimulated with IL$1 \beta$ used as a positive control for LCN2 production and $\beta$-actin as a loading control. E Western blot of liver lysates showed higher amounts of MPO protein in wild type animals treated with oil and $\mathrm{CCl}_{4}$ compared to $\mathrm{Lcn} 2^{-1-}$. Mice injected with LPS for $6 \mathrm{~h}$ were used as positive controls. Mineral oil and $\mathrm{CCl}_{4}$ induced LCN2 production with GAPDH as loading controls. F MPO and LCN2 Western blots were quantified and confirmed significantly lower MPO levels in $\mathrm{LCn}^{-/-}$, while the wild type mice receiving oil showed higher amounts of LCN2 compared to the $\mathrm{CCl}_{4}$ group.

were double the amounts found in $\mathrm{CCl}_{4}$ compared to mineral oil injection (Fig. 1C). By contrast, serum LCN2 as assessed by Western blot analysis showed relatively higher oil injection in correlation to liver mRNA and protein levels (Fig. 1D). These findings indicate that besides NPC, the hepatic parenchymal cells such as hepatocytes produced and secreted LCN2 into circulation as shown in immunofluorescent staining of LCN2 positive hepatocytes in the oil and $\mathrm{CCl}_{4}$ groups (Fig. 1E). 
A

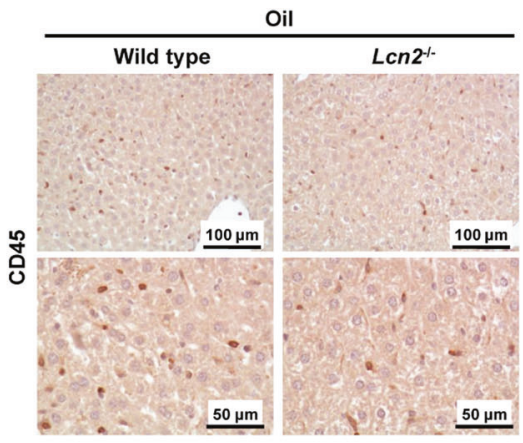

B
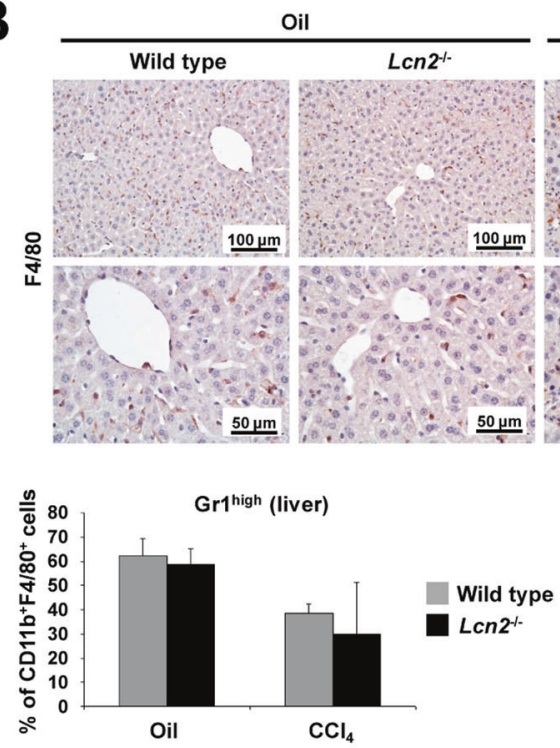

$\mathrm{Cl}_{4}$
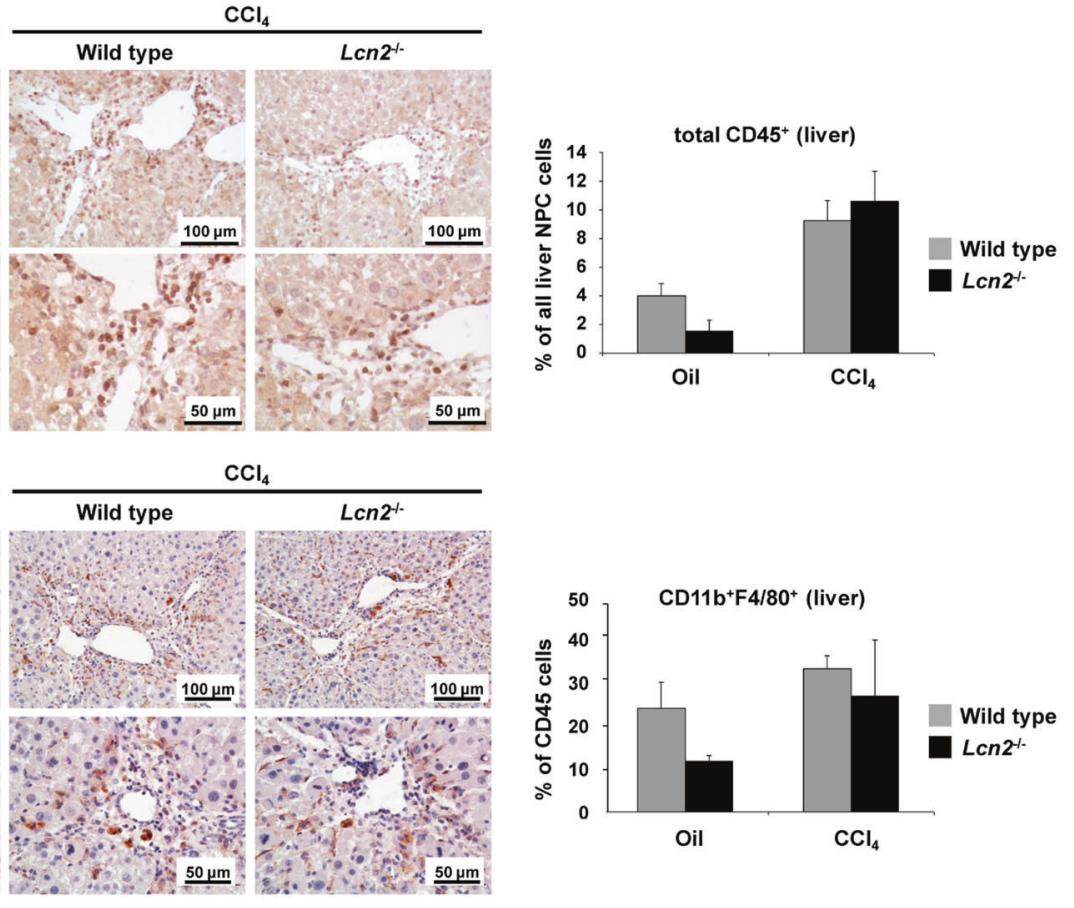

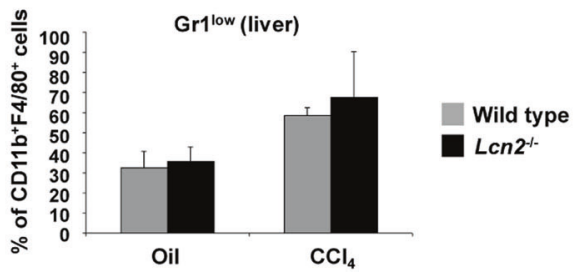

Fig. 4 LCn2 null decreased CD45 ${ }^{+}$and CD11 $\mathbf{b}^{+} \mathbf{F 4 / 8 0 ^ { + }}$ cells in livers of chronic mineral oil. A IHC staining of CD45 ${ }^{+}$and hepatic leukocyte FACS shows significant increases in mice treated with $\mathrm{CCl}_{4}$, but only a marked reduction in $L \mathrm{cn} 2^{-1-}$ mice receiving oil compared to the wild type and no difference between $\mathrm{CCl}_{4}$ treated groups. B IHC staining of F4/80 in step with FACS analysis found lesser amounts of CD11 $\mathrm{b}^{+} \mathrm{F} 4 /$ $80^{+}$in $\mathrm{LCn} 2^{-1-}$ mice receiving oil compared to the wild type with no differentiation between $\mathrm{CCl}_{4}$ wild type mice and $\mathrm{Lcn2} 2^{-1-}$. Mineral oil administration showed high proportions of $\mathrm{Gr}^{\text {high }}$ cells, while $\mathrm{CCl}_{4}$ treated mice showed high proportions of $\mathrm{Gr} 1^{\text {low }}$ cells.

\section{Repeated mineral oil injection-induced hepatic neutrophil infiltration}

Repeated mineral oil i.p. injection-induced visible peritoneal granuloma (data not shown), while immunohistochemistry (IHC) and fluorescent staining showed LCN2 expression in liver NPC. We performed double staining of (i) LCN2 with lymphocyte antigen 6 complex locus G6D (Ly6G), expressed in myeloid-derived cells such as monocytes, granulocytes, and neutrophils, (ii) LCN2 with myeloperoxidase (MPO) that is abundant in neutrophils, and (iii) LCN2 with F4/80, a marker of Kupffer cells, monocytes, and macrophages. LCN2 double staining showed to co-localize with Ly6G and MPO in oil-injected livers, but only partially colocalization in $\mathrm{CCl}_{4}$ groups, while no co-localization of LCN2 with F4/80 (Fig. 2A-C) was found, indicating that chronic injection of both oil and $\mathrm{CCl}_{4}$ induced neutrophil infiltration and activated LCN2 production. Interestingly, significant co-localization of MPO and F4/80 along fibrotic central areas in the liver of animals that received $\mathrm{CCl}_{4}$ were noticed (Fig. 2D), possibly indicating that engulfed macrophages contained MPO.

Lcn2 null decreased neutrophil infiltration after both mineral oil and $\mathrm{CCl}_{4}$ administration as compared to the wild type mice Several publications state LCN2 to promote liver injury and inflammation in alcoholic steatohepatitis and nonalcoholic steatohepatitis (NASH) ${ }^{8-10}$ and LCN2 deficiency to reduce the migration of neutrophils ${ }^{5}$. We stained MPO, the specific marker of neutrophils, and found wild type livers to contain significantly more MPO-positive cells in both oil and $\mathrm{CCl}_{4}$ groups (Fig. 3A-C). MPO expression is restricted to myeloid cells and is synthesized and stored in azurophilic (primary) granules of neutrophils during promyelocytes ${ }^{29}$. To verify the MPO antibody using for its suitability in Western blot, we used extracts from the HL-60 cell line, a promyelocytic cell line derived from human leukemia ${ }^{30}$, and acute promyelocytic leukemia $\mathrm{NB}^{31}$ as positive controls (Fig. 3D). Under basic conditions, these cell lines lacked LCN2, while incubation with lipopolysaccharides (LPS) or LPS and Tunicamycin (TM) induced expression of LCN2, because LCN2 is produced in later stages of neutrophil maturation and stored in specific secondary granules. Western blot analysis of liver lysates confirmed higher MPO protein levels in wild types compared to the Lcn2 null mice. In this analysis liver extracts isolated from LPS-injected mice containing high numbers of activated neutrophils directing LCN2 expression were used as positive controls (Fig. 3D, E).

Lcn2 null decreased $\mathrm{CD}_{4} 5^{+}$and $\mathrm{CD} 11 \mathrm{~b}^{+} \mathrm{F4} / 80^{+}$cells in livers after chronic mineral oil administration but not after chronic $\mathrm{CCl}_{4}$ challenge

We next analyzed the panel of other inflammatory infiltrating cells in livers of wild type and LCn2 null animals through IHC and FACS and found significantly decreased amounts of CD45 ${ }^{+}$leukocytes, $\mathrm{CD} 11 \mathrm{~b}^{+} \mathrm{F} 4 / 80^{+}$monocytes, and macrophages in chronic mineral oil administered Lcn2 null livers compared to wild type, but no difference between the two $\mathrm{CCl}_{4}$ groups (Fig. 4A, B). Interestingly, 
A
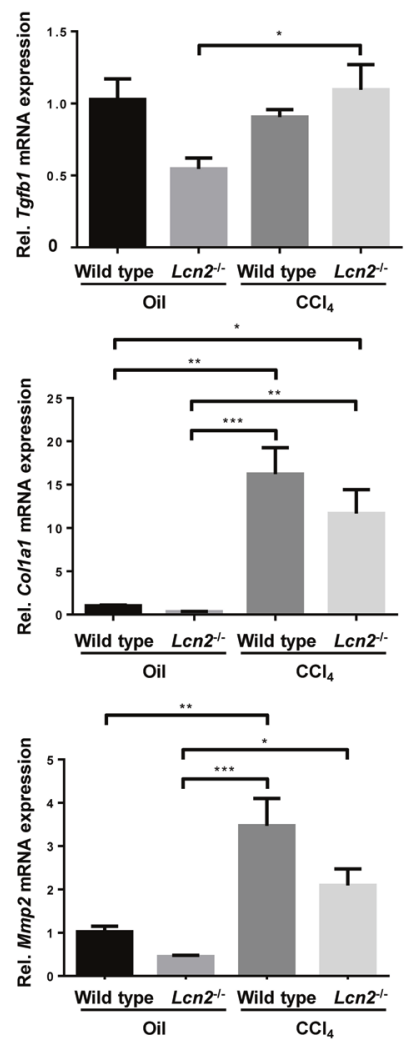

B
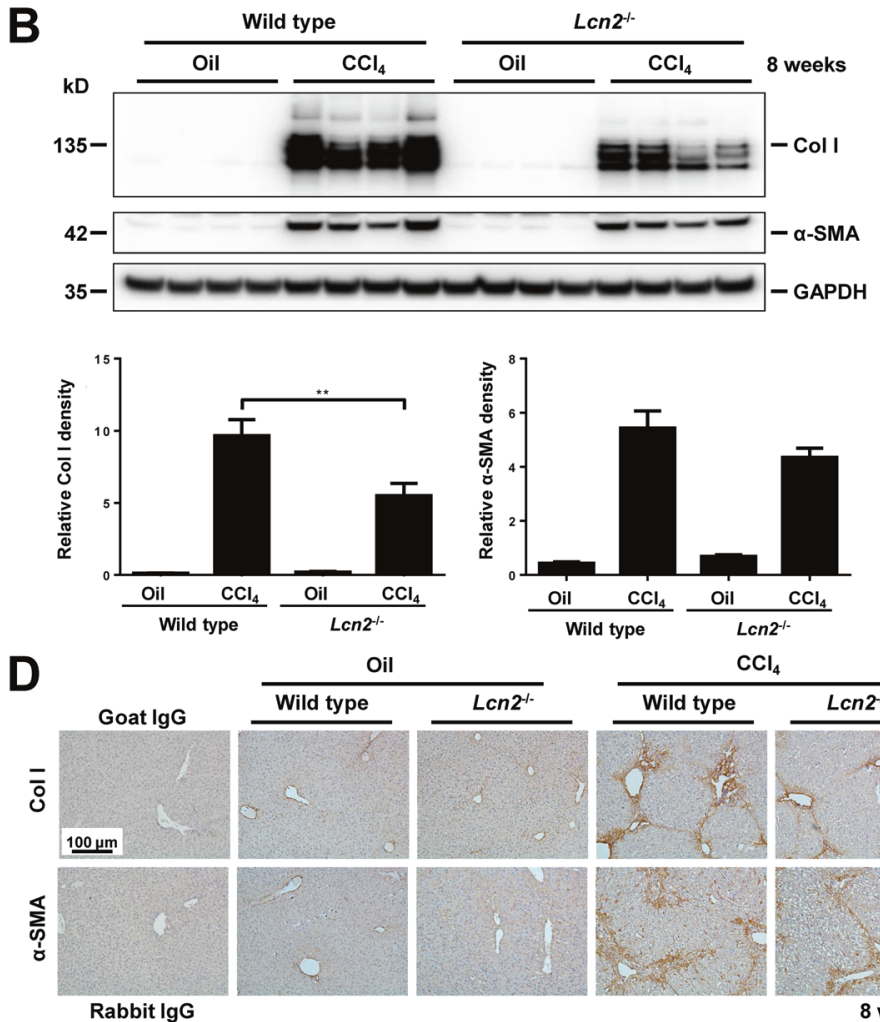

Oil
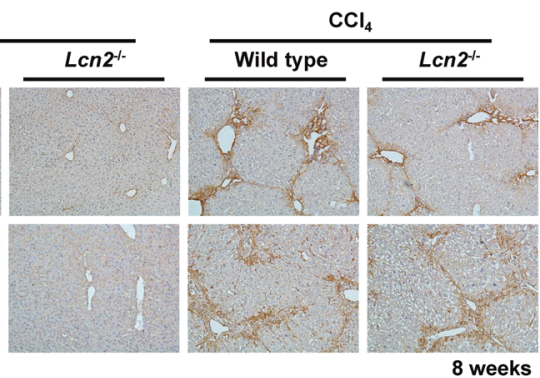
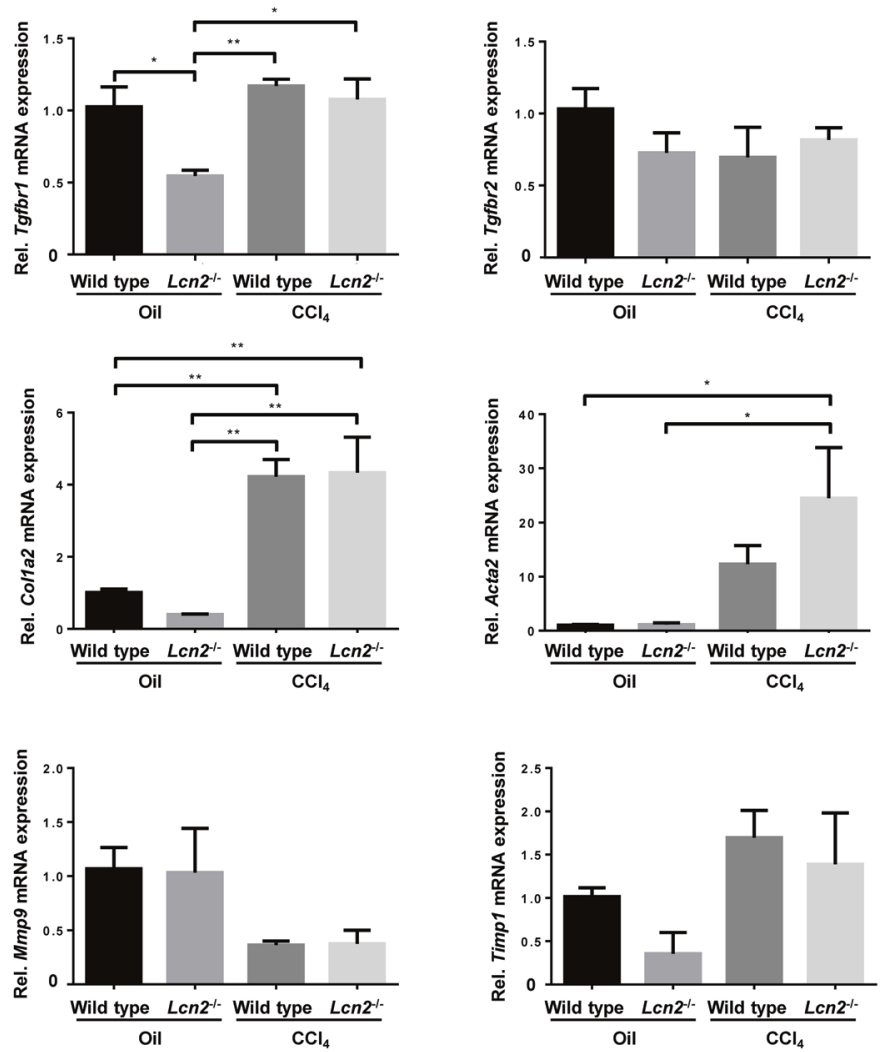

C

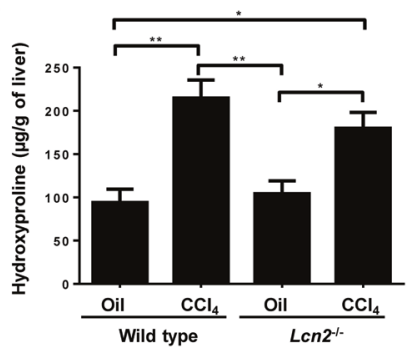

E
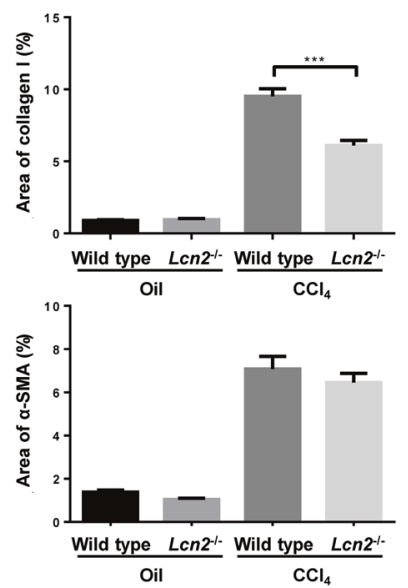

monocytes and macrophages in oil-injected livers showed higher amounts of the $\mathrm{Gr}^{\text {high }}$ monocytes, indicating inflammatory responses. By contrast, the $\mathrm{CCl}_{4}$ livers showed high proportions of $\mathrm{Gr}^{\text {low }}$ monocytes, pointing at chronic wound healing responses, resulting in liver fibrosis.
Lcn2 null decreased inflammatory cytokines and chemokines production in repeated mineral oil injection

Since macrophages are the primary producers of cytokines and chemokines, the cytokine and chemokine mRNA levels correlated well with the numbers of monocytes and macrophages. 
Fig. 5 Lcn2 null decreased collagen accumulation in chronic $\mathbf{C C l}_{\mathbf{4}}$ induced fibrosis. A Quantitative RT-PCR of fibrogenic genes such as $T g f b 1$,

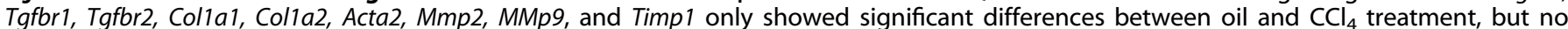
difference between wild type and Lcn2 null animals. B Western blot of liver lysates shows significantly higher amounts of collagen type 1 in $\mathrm{CCl}_{4}$ treated wild type mice as compared to the $\mathrm{LCn} 2^{-1-}$ and no detection in oil application groups. No significant difference of $\alpha$-SMA between wild type and $L C n 2^{-/-}$mice. GAPDH was used as a loading control. The relative Western blot density is shown below. C Hydroxyproline assays show significant levels between different groups, but only slightly lower in $\mathrm{Lcn}^{-1-}$ mice receiving CCl $_{4} \mathrm{Compared} \mathrm{to}^{-1}$ the wild type. D IHC staining of collagen type 1 and $\alpha$-SMA shows slightly less collagen deposition in $L_{c n}{ }^{-1-}$ liver treated with $\mathrm{CCl}_{4} \mathrm{Compared}$ to the wild type and very minimal collagen in oil application groups. Also, a significant increase of $\alpha-\mathrm{SMA}_{\mathrm{A}} \mathrm{CCl}{ }_{4}$ treated livers, but no difference between wild type and $\mathrm{LCn} 2^{-1-}$ mice. Oil application groups showed only very minimal $\alpha$-SMA expression. E Areas of collagen type 1 and $\alpha$-SMA deposition were quantified, and only collagen type 1 was significantly higher in the wild type treated $\mathrm{CCl}_{4}$ compared to the $L \mathrm{Cn} 2^{-/-}$and no difference of $\alpha$-SMA between wild type and $L \mathrm{Cn} 2^{-1-}$ mice.

Only oil-injected Lcn2 null livers showed lesser amounts compared to wild type, while no differences were observed between $\mathrm{CCl}_{4}$ wild type and Lcn2 null (Supplementary Fig. 1). The M1 macrophage marker Nos2 was significantly higher in both $\mathrm{CCl}_{4}$ treated groups, while the M2 polarization marker Arg1 was similar in all groups (Supplementary Fig. 1). These findings indicate that Lcn2 null does affect the inflammatory responses, but not directly in wound healing and fibrogenesis.

\section{Chronic mineral oil application in wild type mice induced inflammatory leukocyte markers}

To confirm that mineral oil application induced liver inflammation, we compared chronic mineral oil-injected mice with sham operation and found similar amounts of hepatic Cd45 mRNA, the marker of total leukocytes, but the mRNA levels such as $C d 11 b$, F4/80, Ly6G, Mpo and Lcn2, indicators of inflammatory responses, were significantly higher in the wild type receiving mineral oil (Supplementary Fig. 2A). IHC staining confirmed increased numbers of F4/80, MPO, and LCN2 positive cells in mineral oilinjected wild type livers (Supplementary Fig. 2B). In addition, the mRNA of inflammatory cytokines, chemokines, and their receptors such as $\|11 a\| 11 b,, \| 1 r 1, C \mathrm{Cl}$, Tnfa, and Tnfr1 were significantly upregulated in wild type animals receiving eight-week oil injection. The Mmp9 gelatinase produced in neutrophils and Timp1 expression were both increased in wild type oil administered mice, while Mmp2 were comparable (Supplementary Fig. 2C).

\section{Len2 null slightly diminished collagen accumulation in chronic $\mathrm{CCl}_{4}$-induced fibrosis}

Although the hepatic leukocyte population assessed by FACS showed no significant difference of liver inflammatory cells between wild type and $\mathrm{LCn}^{-1-}$ in $\mathrm{CCl}_{4}$-treated groups (Fig. 4), Lcn2 null mice showed lower numbers of MPO positive cells and lower levels of MPO protein (Fig. 3). With regard to liver fibrosis, we found no difference in Tgfb1, Tgfbr1, Tgfbr2, Mmp2, Mmp9, Timp1, Col1a1, and Acta2 (a-Sma) mRNA between wild type and Lcn2 ${ }^{-1-}$ mice receiving $\mathrm{CCl}_{4}$ (Fig. 5A), but wild type mice developed slightly more liver fibrosis than $L C n 2^{-/-}$mice as evidenced by higher levels of collagen type I protein in Western blot (Fig. 5B). Measurement of hydroxyproline, a major component of the collagen protein and collagen type I IHC duly confirmed higher levels of fibrosis in wild type mice compared to the $L c n 2^{-1-}$. No significant difference was observed in a-SMA mRNA and protein levels and no liver fibrosis in mineral oil administrations (Fig. 5C-E). RNA-Seq confirmed downregulation of collagen-containing extracellular matrix in $\mathrm{CCl}_{4}$ administered Lcn2 null compared to the wild type mice (Fig. 6, Supplementary Figs. 3-5).

\section{Chronic mineral oil administration suppressed gene sets involved in myeloid leukocyte activation and immune responses in Lcn2 null mice}

Granulocytes and monocytes/macrophages are myeloid lineage cells and are mainly responsible for innate immune responses. We found reduced amounts of liver MPO in IHC staining and Western blot analysis in Lcn2 null compared to the wild type. Additionally, characterization of liver leukocytes by FACS also showed significantly lower amounts of $\mathrm{CD} 11 \mathrm{~b}^{+} \mathrm{F} 4 / 80^{+}$monocytes/ macrophages in chronic mineral oil administered Lcn2 null mice compared to the wild type. RNA-Seq results supported our findings that $L c n 2$ null livers decreased expression of the gene sets involved in myeloid leukocyte activation, especially neutrophil and monocytes/macrophage functions such as leukocyte migration and cell adhesion as shown in the heat maps (Fig. 6, Supplementary Figs. 3-5).

\section{Len2 null mice increased lipid biosynthesis}

Both mineral oil and $\mathrm{CCl}_{4}$ administration did enrich genes involved in lipid biosynthetic and metabolic process such as cholesterol biosynthesis in chronic $\mathrm{CCl}_{4}$ application. We found more lipid droplets in both mineral oil and $\mathrm{CCl}_{4}$ application in $\mathrm{Lcn} 2$ null compared to wild type mice (data not shown).

\section{Chronic application of $\mathrm{CCl}_{4}$ induced liver cell proliferation in} Len2 null more than in wild type mice

Surprisingly, RNA-Seq showed significantly enriched gene sets involved in DNA replication and cell cycle in $\mathrm{LCn} 2^{-/-}$mice receiving eight week $\mathrm{CCl}_{4}$ compared to wild type controls (Fig. 6, Supplementary Figs. 3-5). These findings were further confirmed by increased cyclin D1, phosphorylated retinoblastoma protein $(\mathrm{Rb})$, and proliferating cell nuclear antigen (PCNA) in Western blots, while showing no significant differences in cyclin $E, C D K 2$, and cyclin A (Fig. 7A and Supplementary Fig. 6). The antigen identified by monoclonal antibody Ki67 in IHC staining showed significantly increased numbers of Ki67 positive hepatocytes in zone 3 livers of $L c n 2$ null compared to the wild type mice (Fig. 7B).

\section{DISCUSSION}

Upon eight week-mineral oil and $\mathrm{CCl}_{4}$ application, we unexpectedly found upregulation of $L c n 2$ expression in both groups of wild type animals, but no expression in Lcn2 null mice. Levels of Lcn2 mRNA and LCN2 protein were significantly higher in mineral oil administered livers. LCN2 IHC staining identified strongly positive NPC, while LCN2 positive staining in $\mathrm{CCl}_{4}$ liver was detected both in NPC and hepatocytes around the central veins. LCN2 positive NPC of mineral oil animals was scattered homogeneously throughout the liver but the numbers of cells were significantly lower than in the $\mathrm{CCl}_{4}$ treated group. These discrepancies most likely indicate that other cell types such as Kupffer cells and hepatocytes produced LCN2 as well. Especially hepatocytes are the major cell type that produced serum LCN2 in the models of bacterial infection or partial hepatectomy ${ }^{11}$.

Mouse neutrophils are commonly identified based on the cell surface expression of Ly6G and CD11b with the cytoplasmic marker MPO. We performed double staining of LCN2 with Ly6G and LCN2 with MPO and identified the LCN2 positive NPC in oiltreated livers as neutrophils. Neutrophil LCN2 is produced during band cells and segmented cells development and is known as a secondary granule protein ${ }^{32-34}$. Therefore, IHC staining showed 

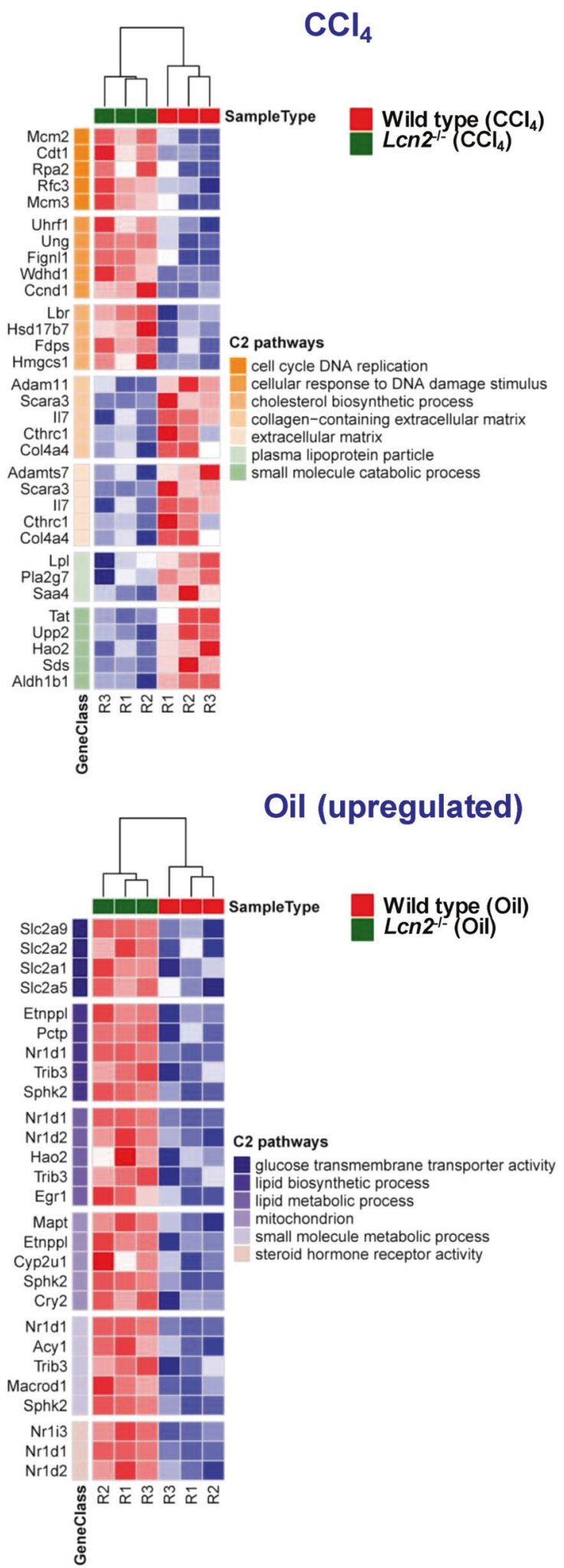

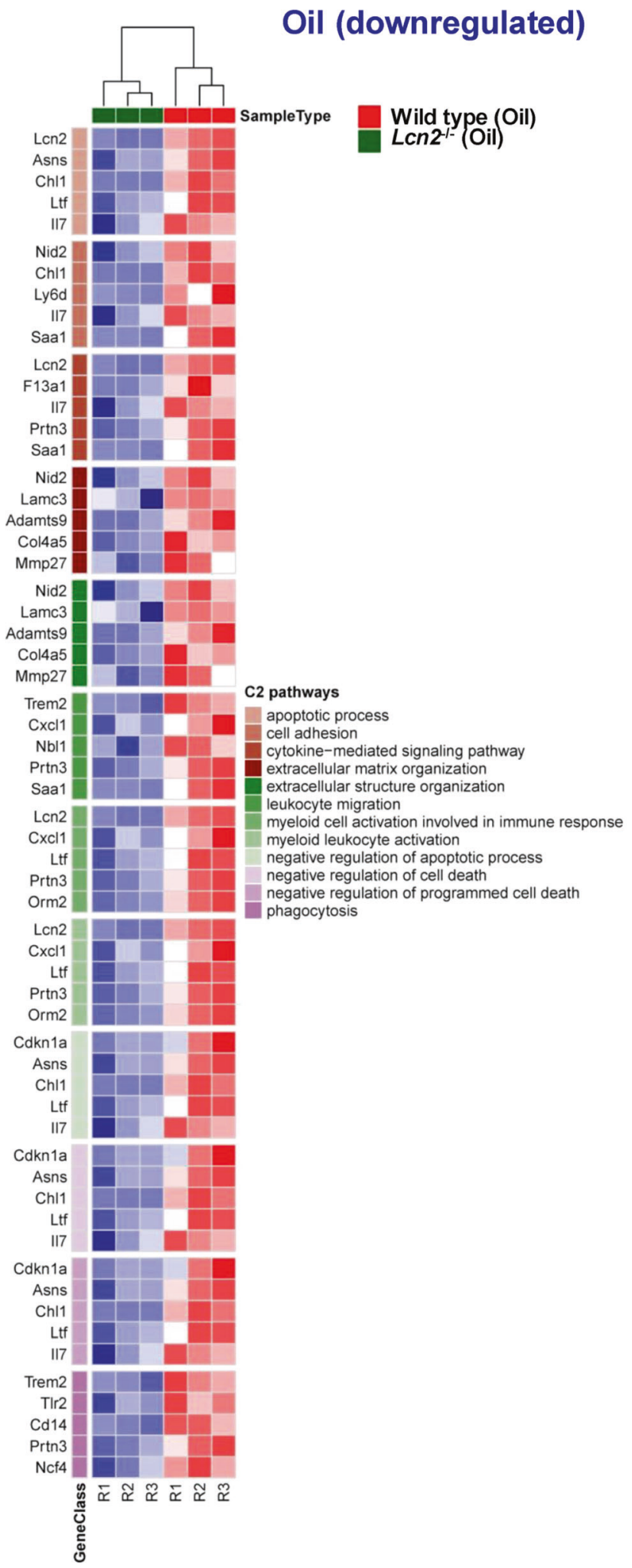

Fig. 6 Go heatmaps. Lcn2 null and wild type mice were compared in chronic $\mathrm{CCl}_{4}$ or mineral oil administered groups. The top five genes per gene set were adjusted to the $p$ values $\leq 0.05$ and gene sorting by Log 2 of fold change. Upregulation and downregulation of the gene sets and the compatible pathways as shown.

LCN2 positive very strongly in neutrophils as compared to the LCN2 in hepatocytes and other cell types such as macrophages, which secrete LCN2 by classical pathways ${ }^{35}$. This explains why the levels of serum LCN2 are significantly higher in wild type animals receiving mineral oil administration than in the fibrotic $\mathrm{CCl}_{4}$ group. Intraperitoneal injection of mineral oil leads to the formation of lipo-granulomas of peritoneum and mesenteric lymph nodes consisting of phagocytic cells engulfed the oil droplets ${ }^{36}$. 
A

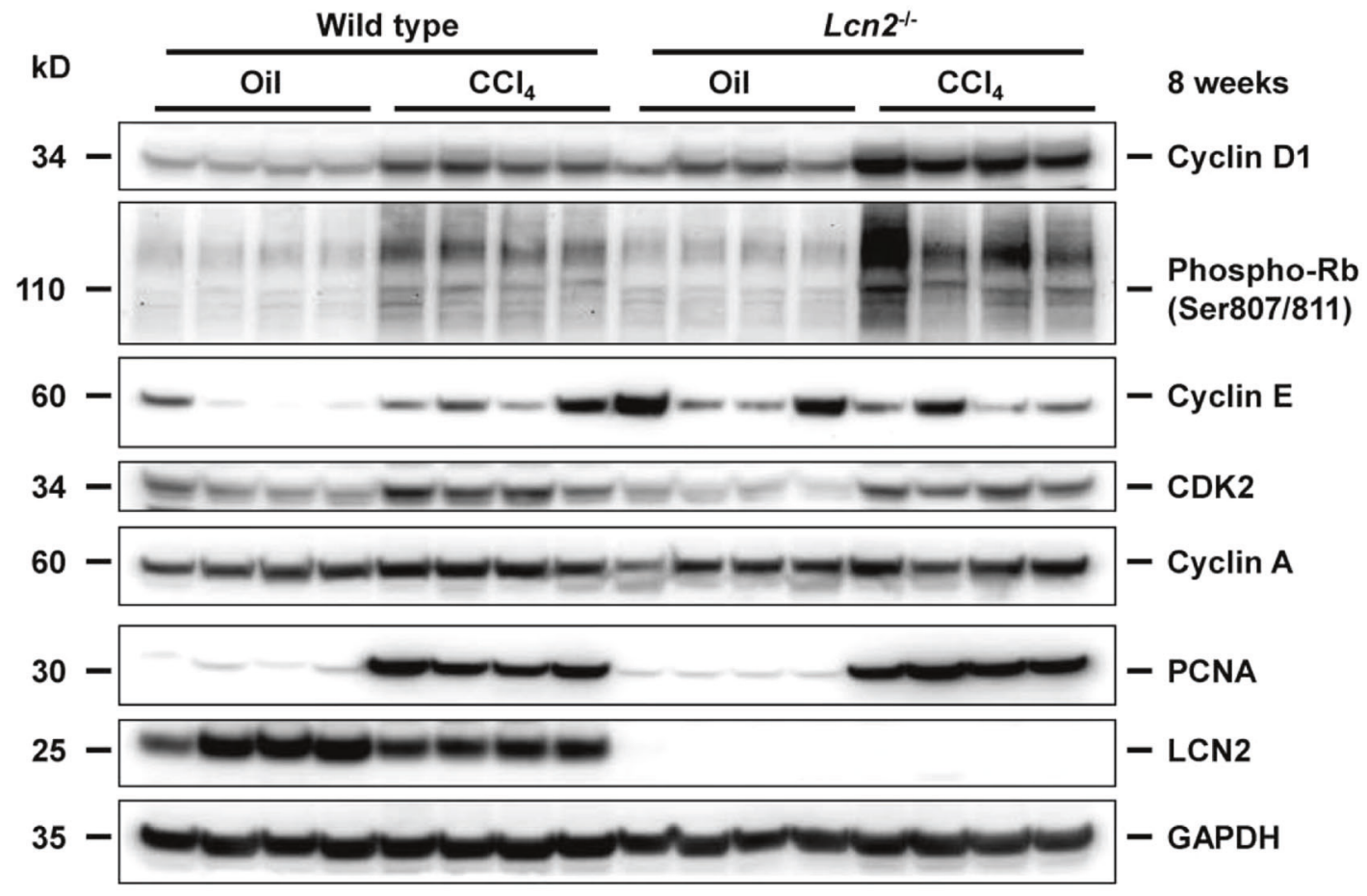

B
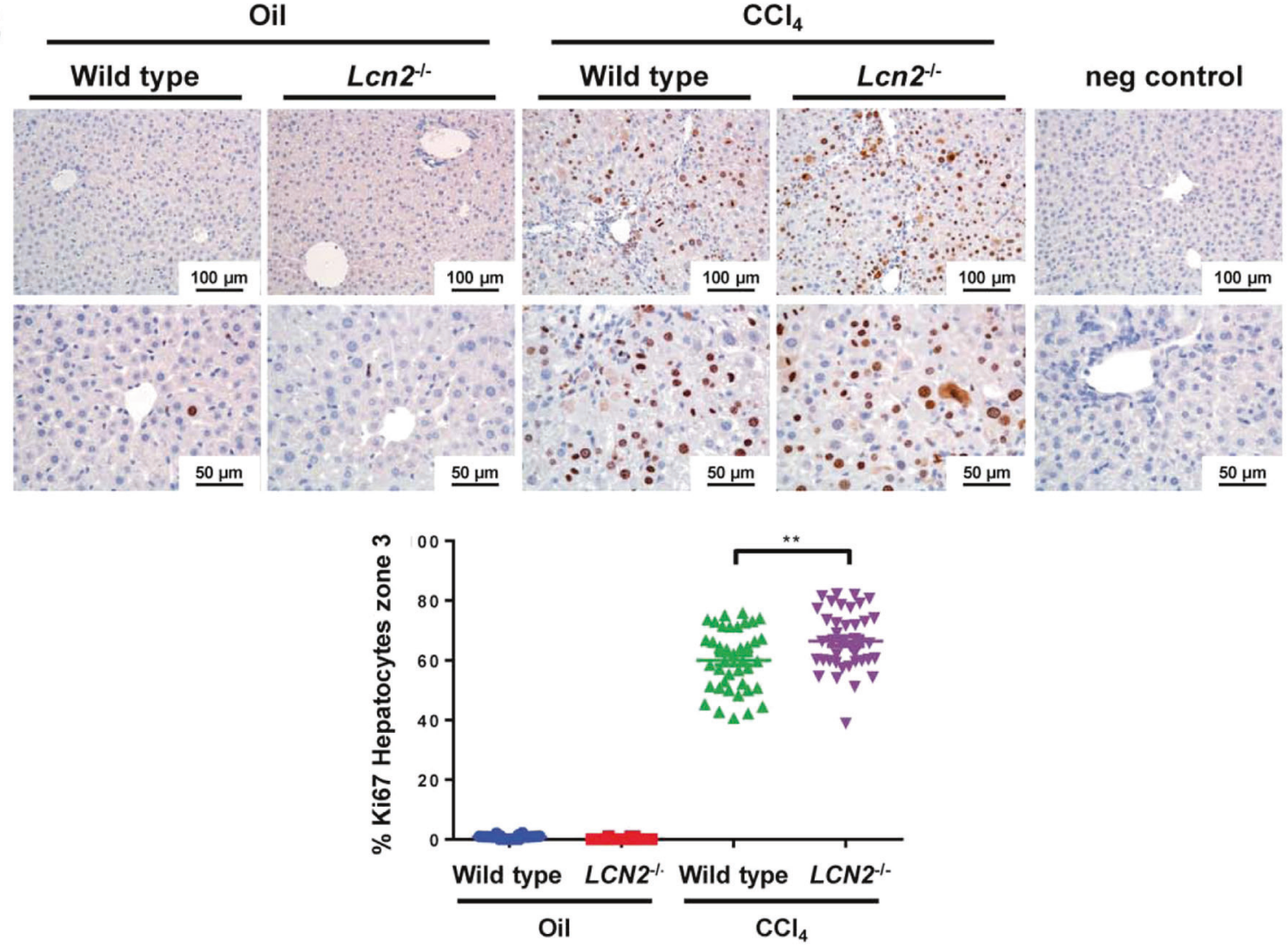

Fig. 7 Chronic $\mathrm{CCl}_{4}$ application induced liver cell proliferation. A Western blots of proteins involved in cell cycle and cell proliferation markers such as cyclin D1, phosphorylated-retinoblastoma (Rb), cyclin E, CDK2, cyclin A, PCNA, and LCN2 as depicted. GAPDH was used as a loading control, and the quantification of Western blots is shown in Supplementary Fig. 6 . B IHC showed a significant increase in Ki67 positive hepatocyte nuclei in liver zone 3 of the Lcn2 null compared to the wild type mice. 
Kupffer cells, the residential macrophages, are the majority of these phagocytic cells in the liver. Upon macrophage activation, they produce cytokines and chemokines attracting neutrophil infiltration as shown in our chronic 8 week-oil administration experiment. These findings are in line with a report from Lee et al. demonstrating that exposure to hydrocarbon oils is associated with the development of chronic inflammation and modulates neutrophil recruitment in peritoneal fluid through IL-1a and IL-1R signaling in a CXCR2-dependent manner ${ }^{37}$. We also found significant mRNA upregulation of several inflammatory cytokines and chemokines including $\|1 a\| ,1 \beta$, Tnf- $a, \| 6, C c l 2, C x C l 1, C x C l 2$, $\mathrm{C} x \mathrm{Cl} 5$ and their receptor mRNAs in chronic mineral oil application of wild type mice.

The inflammatory responses to chronic $\mathrm{CCl}_{4}$ intoxication using the mineral oil vehicle as a control, showed mineral oil alone to induce more hepatic inflammation than expected and more severe in wild type than $\mathrm{LCn} 2^{-1-}$ mice. We found no significant differences between wild type and $L c n 2^{-1-}$ receiving $\mathrm{CCl}_{4}$ groups, except for the amounts of $\mathrm{MPO}^{+}$cell counts via IHC and Western blot analysis. These findings indicate that combinations of hepatocyte injuries, chronic oxidative stress from $\mathrm{CCl}_{4}$, together with hepatocyte- necrosis, apoptosis, proliferation, and tissue repair lead to liver fibrosis. This process may obscure the differences in inflammatory responses between wild type and $L \mathrm{Cn} 2^{-1-}$ mice. MPO is expressed by circulating neutrophils and monocytes/macrophages in the human liver ${ }^{38}$. We found a 56-kD protein, the predicted molecular mass of MPO (heavy chain) in liver extracts to be comparable between mineral oil- and $\mathrm{CCl}_{4}$ injected wild type with similar lower values in $\mathrm{Lcn} 2^{-/-}$liver after both oil and $\mathrm{CCl}_{4}$ administration. Immunofluorescence double staining of liver slides demonstrated co-localization of MPO with LCN2 and LCN2 with Ly6G in mineral oil application neutrophils, while the $\mathrm{MPO}^{+}$cells found along the fibrotic septa in $\mathrm{CCl}_{4}$ livers were not all co-localized with LCN2 and Ly6G, but with F4/80 instead. By contrast, we found no co-localization of LCN2 with $\mathrm{F} 4 / 80$, but instead $\mathrm{MPO}^{+}$cells after chronic mineral oil application in both spindle shape (macrophages) and round segmental polymorphonuclear cells located in the sinusoids (Supplementary Fig. 2B). MPO is synthesized during promyelocytes and promyelomonocytes in the bone marrow and terminated as soon as myeloid progenitors enter the myelocyte stage of differentiation $^{29}$. Mature monocytes contain a small size peroxidase in primary granules ${ }^{39}$. The co-localization of MPO and F4/80 cells along the fibrotic central veins is most likely lipid-laden foam cell macrophages engulfed the MPO oxidized low-density lipoprotein (LDL) from $\mathrm{CCl}_{4}$ intoxication, which is similar in macrophages at human atherosclerotic lesions ${ }^{40}$.

For $\mathrm{CCl}_{4}$-induced liver fibrosis, we found only the significantly increased fibrogenic mRNA gene expression in $\mathrm{CCl}_{4}$ treated mice compared to oil administration. Surprisingly, the collagen protein deposition was significantly higher in $\mathrm{CCl}_{4}$ treated wild type mice compared to the $L C n 2^{-1-}$, as evidenced by Western blot analysis, larger areas stained positive for collagen type 1, and higher levels of hydroxyproline in liver tissue. These findings seem to correspond well with the levels of MPO protein in Western blot and IHC. In line, RNA-Seq results confirmed that the $L \mathrm{Cn} 2^{-/-}$mice provoke defects in gene sets involved in myeloid leukocyte function, especially neutrophil activation during immune responses including leukocyte migration, cell adhesion and neutrophil degranulation. Additionally, $L C n 2^{-/-}$mice decreased the expression of genes involved in cell surface receptor signaling, cellular response to cytokine stimulus and cytokine-mediated signaling pathways. For this reason, we found high levels of inflammatory cytokines and chemokines in wild type mice receiving oil compared to the $L c n 2^{-/-}$mice (Supplementary Fig. 1).

The effects of LCN2 and liver cell proliferation are still controversial. One particular example of a partial hepatectomy model of both hepatocyte-specific knockout (i.e. $L \mathrm{cn} 2^{\text {Hep-l-}}$ ) and
$\mathrm{LCn} 2^{-/-}$mice showed lower rates of liver regeneration, compared to the wild type ${ }^{11}$, while others found that LCN2 does not to alter hepatocyte regeneration ${ }^{41}$. It was the first time in our chronic $\mathrm{CCl}_{4}$ model that we found distinct upregulation of gene sets involved in DNA replication and cell cycle in livers of $L c n 2^{-/-}$mice receiving $\mathrm{CCl}_{4}$ compared to the wild type counterparts (Fig. 6). The cell cycle-regulated proteins such as cyclin D1 and phosphorylated retinoblastoma were significantly higher in $L c n 2^{-/-}$livers. In our recent report, we found $\mathrm{LCn} 2^{-/-}$mice to develop more hepatocyte apoptosis after chronic $\mathrm{CCl}_{4}$ application compared to the wild type ${ }^{21}$. This hepatocyte damage may enhance compensating hepatocyte proliferation in $\mathrm{LCn}^{-1-}$ mice, as indicated by increased PCNA expression in Western blot and the numbers of Ki67-positive cells in liver zone 3, the most severe hepatocyte injured areas (Figs. 7A and B). The ability of hepatocyte regeneration combined with lower leukocyte inflammatory responses might promote tissue repair and attenuate liver fibrosis in $\mathrm{LCn}^{-/-}$mice. Furthermore, $\mathrm{Lcn} 2^{-/-}$mice also downregulated gene sets for collagen-containing extracellular matrix structure organization in both oil and $\mathrm{CCl}_{4}$ application compared to the wild type counterparts.

In summary, chronic mineral oil intraperitoneal administration induced hepatic inflammation through innate immune responses via myeloid cell activation. The Lcn2 null mice developed less inflammation due to defects in genes involved in the myeloid cell activation process, and downregulated gene sets for collagencontaining extracellular matrix leading to mitigation of the liver fibrosis. Additionally, it is the first time that we found Lcn2 null mice to show enrichment of genes responsible for DNA damage and cell cycle DNA replication, compared to the wild type mice upon chronic $\mathrm{CCl}_{4}$ liver injury.

\section{DATA AVAILABILITY}

The datasets used and/or analyzed during the current study are available from the corresponding author on reasonable request. Expression data is accessible via the Gene Expression Omnibus (GEO) under accession number GSE176093.

\section{REFERENCES}

1. Kjeldsen, L., Johnsen, A. H., Sengelov, H. \& Borregaard, N. Isolation and primary structure of NGAL, a novel protein associated with human neutrophil gelatinase. $J$ Biol Chem 268, 10425-10432 (1993).

2. Goetz, D. H. et al. The neutrophil lipocalin NGAL is a bacteriostatic agent that interferes with siderophore-mediated iron acquisition. Mol Cell 10, 1033-1043 (2002).

3. Flo, T. H. et al. Lipocalin 2 mediates an innate immune response to bacterial infection by sequestrating iron. Nature 432, 917-921 (2004).

4. Berger, T. et al. Lipocalin 2-deficient mice exhibit increased sensitivity to Escherichia coli infection but not to ischemia-reperfusion injury. Proc Natl Acad Sci U S A 103, 1834-1839 (2006).

5. Schroll, A. et al. Lipocalin-2 ameliorates granulocyte functionality. Eur J Immunol 42, 3346-3357 (2012).

6. Liu, Z., Petersen, R. \& Devireddy, L. Impaired neutrophil function in $24 \mathrm{p} 3$ null mice contributes to enhanced susceptibility to bacterial infections. J Immunol 190, 4692-4706 (2013).

7. Wang, Q. et al. Lipocalin 2 protects against Escherichia coli infection by modulating neutrophil and macrophage function. Front Immunol 10, 2594 (2019).

8. Ye, D. et al. Lipocalin-2 mediates non-alcoholic steatohepatitis by promoting neutrophil-macrophage crosstalk via the induction of CXCR2. J Hepatol 65, 988-997 (2016).

9. Wieser, V. et al. Lipocalin 2 drives neutrophilic inflammation in alcoholic liver disease. J Hepatol 64, 872-880 (2016).

10. Cai, Y. et al. The detrimental role played by lipocalin-2 in alcoholic fatty liver in mice. Am J Pathol 186, 2417-2428 (2016).

11. $\mathrm{Xu}, \mathrm{M}$. J. et al. Liver is the major source of elevated serum lipocalin-2 levels after bacterial infection or partial hepatectomy: a critical role for IL-6/STAT3. Hepatology 61, 692-702 (2015).

12. Cramer, E. P. et al. Lipocalin-2 from both myeloid cells and the epithelium combats Klebsiella pneumoniae lung infection in mice. Blood 129, 2813-2817 (2017). 
13. Li, H. et al. Hepatocytes and neutrophils cooperatively suppress bacterial infection by differentially regulating lipocalin-2 and neutrophil extracellular traps. Hepatology 68, 1604-1620 (2018).

14. Srinivasan, G. et al. Lipocalin 2 deficiency dysregulates iron homeostasis and exacerbates endotoxin-induced sepsis. J Immunol 189, 1911-1919 (2012).

15. Borkham-Kamphorst, E. et al. Protective effects of lipocalin-2 (LCN2) in acute liver injury suggest a novel function in liver homeostasis. Biochim Biophys Acta 1832, 660-673 (2013)

16. Ariza, X. et al. Neutrophil gelatinase-associated lipocalin is a biomarker of acuteon-chronic liver failure and prognosis in cirrhosis. $J$ Hepatol 65, 57-65 (2016).

17. Ye, Z. et al. Serum lipocalin-2, cathepsin $S$ and chemerin levels and nonalcoholic fatty liver disease. Mol Biol Rep 41, 1317-1323 (2014).

18. Chen, J. et al. Hepatic lipocalin 2 promotes liver fibrosis and portal hypertension. Sci Rep 10, 15558 (2020).

19. Meier, E. M. et al. Circulating lipocalin 2 is neither related to liver steatosis in patients with non-alcoholic fatty liver disease nor to residual liver function in cirrhosis. Cytokine 85, 45-50 (2016).

20. Karlmark, K. R. et al. Hepatic recruitment of the inflammatory Gr1+ monocyte subset upon liver injury promotes hepatic fibrosis. Hepatology 50, 261-274 (2009).

21. Borkham-Kamphorst, E., Haas, U., Van de Leur, E., Trevanich, A. \& Weiskirchen, R. Chronic carbon tetrachloride applications induced hepatocyte apoptosis in Lipocalin 2 null mice through endoplasmic reticulum stress and unfolded protein response. Int J Mol Sci 21, 5230 (2020).

22. Schmittgen, T. D. \& Livak, K. J. Analyzing real-time PCR data by the comparative CT method. Nat Protoc 3, 31101-31108 (2008).

23. Patel, H. et al. (2021, May 13). nf-core/rnaseq: nf-core/rnaseq v3.1 - Lead Alligator. Available from https://doi.org/10.5281/zenodo.4757022.

24. Daley, T. \& Smith, A. D. Predicting the molecular complexity of sequencing libraries. Nat Methods 10, 325-327 (2013).

25. Dobin, A. et al. STAR: ultrafast universal RNA-seq aligner. Bioinformatics 29, 15-21 (2013).

26. Patro, R., Duggal, G., Love, M.I., Irizarry, R.A. \& Kingsford, C. Salmon provides fast and bias-aware quantification of transcript expression. Nat Methods 14, 417-419 (2017).

27. Love, M. I., Huber, W. \& Anders, S. Moderated estimation of fold change and dispersion for RNA-seq data with DESeq2. Genome Biol. 15, 550 (2014).

28. Kolberg, L., Raudvere, U., Kuzmin, I., Vilo, J. \& Peterson, H. gprofiler2 - an R package for gene list functional enrichment analysis and namespace conversion toolset g:Profiler. F1000Res 9, ELIXIR-709 (2020).

29. Hansson, M., Olsson, I. \& Nauseef, W. M. Biosynthesis, processing, and sorting of human myeloperoxidase. Arch Biochem Biophys 445, 214-224 (2006).

30. Koeffler, H. P., Ranyard, J. \& Pertcheck, M. Myeloperoxidase: its structure and expression during myeloid differentiation. Blood 65, 484-491 (1985).

31. Lanotte, $M$. et al. NB4, a maturation inducible cell line with $t(15 ; 17)$ marker isolated from a human acute promyelocytic leukemia (M3). Blood 77, 1080-1086 (1991).

32. Borregaard, N., Sehested, M., Nielsen, B. S., Sengeløv, H. \& Kjeldsen, L. Biosynthesis of granule proteins in normal human bone marrow cells. Gelatinase is a marker of terminal neutrophil differentiation. Blood 85, 812-817 (1995).

33. $\mathrm{Xu}, \mathrm{S}$. Y. et al. Purification and characterization of a human neutrophil lipocalin (HNL) from the secondary granules of human neutrophils. Scand J Clin Lab Invest 54, 365-376 (1994).

34. Sevéus, L., Amin, K., Peterson, C. G., Roomans, G. M. \& Venge, P. Human neutrophil lipocalin (HNL) is a specific granule constituent of the neutrophil granulocyte. Studies in bronchial and lung parenchymal tissue and peripheral blood cells. Histochem Cell Biol 107, 423-432 (1997).

35. Meheus, L. A. et al. Identification by microsequencing of lipopolysaccharideinduced proteins secreted by mouse macrophages. J Immunol 151, 1535-1547 (1993).

36. Shaheen, V. M. et al. Immunopathogenesis of environmentallyinduced lupus in mice. Environ.l Health Perspect 107(Suppl 5), 723-727 (1999).

37. Lee, P. Y. et al. IL-1 a modulates neutrophil recruitment in chronic inflammation induced by hydrocarbon oil. J Immunol 186, 1747-1754 (2011).

38. Brown, K. E., Brunt, E. M. \& Heinecke, J. W. Immunohistochemical detection of myeloperoxidase and its oxidation products in Kupffer cells of human liver. Am J Pathol 159, 2081-2088 (2001).
39. van der Rhee, H. J., de Winter, C. P. \& Daems, W. T. Fine structure and peroxidatic activity of rat blood monocytes. Cell Tissue Res 185, 1-16 (1977).

40. Daugherty, A., Dunn, J. L., Rateri, D. L. \& Heinecke, J. W. Myeloperoxidase, a catalyst for lipoprotein oxidation, is expressed in human atherosclerotic lesions. $J$ Clin Invest. 94, 437-444 (1994).

41. Kienzl-Wagner, K. et al. The role of lipocalin-2 in liver regeneration. Liver Int $\mathbf{3 5}$, 1195-1202 (2015).

\section{ACKNOWLEDGEMENTS}

The authors are grateful to Jens Bauer for help in performing FACS analysis. We thank the Genomics Facility of IZKF Aachen for RNA-Seq library preparation. In addition, the authors thank Dr. Thorsten Berger and Tak W. Mak (The Campbell Family Institute for Breast Cancer Research and Ontario Cancer Institute, University Health Network, Toronto, Ontario, Canada) for providing $L c n 2^{-/-}$mouse. R. W. is supported by grants from the German Research Foundation (WE 2554/13-1, WE 2554/15-1, and WE 2554/ 17-1). E. B.-K. received a grant from the Interdisciplinary Centre for Clinical Research within the Faculty of Medicine at the RWTH Aachen University (IZKF Aachen, project 03-9).

\section{AUTHOR CONTRIBUTIONS}

E.B.-K. and R.W. performed study concept and design. E.B.-K., U.H. and M.P.-S. performed the experiments. A.T.A. has done the bioinformatics for this study. E.B.-K. and R.W. prepared the original draft. All authors read and approved the final paper.

\section{FUNDING}

Open Access funding enabled and organized by Projekt DEAL.

\section{COMPETING INTERESTS}

The authors declare no competing interests.

\section{ADDITIONAL INFORMATION}

Supplementary information The online version contains supplementary materia available at https://doi.org/10.1038/s41374-021-00672-9.

Correspondence and requests for materials should be addressed to Erawan Borkham-Kamphorst or Ralf Weiskirchen.

Reprints and permission information is available at http://www.nature.com/ reprints

Publisher's note Springer Nature remains neutral with regard to jurisdictional claims in published maps and institutional affiliations.

(i) Open Access This article is licensed under a Creative Common cc) Attribution 4.0 International License, which permits use, sharing, adaptation, distribution and reproduction in any medium or format, as long as you give appropriate credit to the original author(s) and the source, provide a link to the Creative Commons license, and indicate if changes were made. The images or other third party material in this article are included in the article's Creative Commons license, unless indicated otherwise in a credit line to the material. If material is not included in the article's Creative Commons license and your intended use is not permitted by statutory regulation or exceeds the permitted use, you will need to obtain permission directly from the copyright holder. To view a copy of this license, visit http://creativecommons. org/licenses/by/4.0/.

(c) The Author(s) 2021 